\title{
MAIT cell alterations in adults with recent-onset and long-term type 1 diabetes
}

\author{
Isabelle Nel ${ }^{1}$ (D) Lucie Beaudoin ${ }^{1} \cdot$ Zouriatou Gouda $^{1} \cdot$ Camille Rousseau $^{1} \cdot$ Pauline Soulard $^{1} \cdot$ Matthieu Rouland $^{1}$ (D) \\ Léo Bertrand ${ }^{1}$ (D) $\cdot$ Christian Boitard ${ }^{1,2} \cdot$ Etienne Larger $^{1,2}$ (D) $\cdot$ Agnès Lehuen ${ }^{1}$ (D)
}

Received: 7 December 2020 / Accepted: 27 April 2021 / Published online: 4 August 2021

(C) The Author(s), under exclusive licence to Springer-Verlag GmbH Germany, part of Springer Nature 2021

\begin{abstract}
Aims/hypothesis Mucosal-associated invariant T (MAIT) cells are innate-like T lymphocytes expressing an $\alpha \beta \mathrm{T}$ cell antigen receptor that recognises the MHC-related 1 molecule. MAIT cells are altered in children at risk for and with type 1 diabetes, and mouse model studies have shown MAIT cell involvement in type 1 diabetes development. Since several studies support heterogeneity in type 1 diabetes physiopathology according to the age of individuals, we investigated whether MAIT cells were altered in adults with type 1 diabetes.

Methods MAIT cell frequency, phenotype and function were analysed by flow cytometry, using fresh peripheral blood from 21 adults with recent-onset type 1 diabetes (2-14 days after disease onset) and 47 adults with long-term disease ( $>2$ years after diagnosis) compared with 55 healthy blood donors. We also separately analysed 17 women with long-term type 1 diabetes and an associated autoimmune disease, compared with 30 healthy women and 27 women with long-term type 1 diabetes.

Results MAIT cells from adults with recent-onset type 1 diabetes, compared with healthy adult donors, harboured a strongly activated phenotype indicated by an elevated CD25 $5^{+}$MAIT cell frequency. In adults with long-term type 1 diabetes, MAIT cells displayed an activated and exhausted phenotype characterised by high CD25 and programmed cell death 1 (PD1) expression and a decreased production of proinflammatory cytokines, IL-2, IFN- $\gamma$ and TNF- $\alpha$. Even though MAIT cells from these patients showed upregulated IL-17 and IL-4 production, the polyfunctionality of MAIT cells was decreased (median 4.8 vs $13.14 \%$ of MAIT cells, $p<0.001$ ) and the frequency of MAIT cells producing none of the effector molecules analysed increased (median $34.40 \mathrm{vs} \mathrm{19.30 \%} \mathrm{of} \mathrm{MAIT} \mathrm{cells,}$ $p<0.01$ ). Several MAIT cell variables correlated with $\mathrm{HbA}_{1 \mathrm{c}}$ level and more particularly in patients with recent-onset type 1 diabetes. In women with long-term type 1 diabetes, MAIT cell alterations were more pronounced in those with an associated autoimmune disease than in those without another autoimmune disease. In women with long-term type 1 diabetes and an associated autoimmune disease, there was an increase in CD69 expression and a decrease in the survival B-cell lymphoma 2 (BCL-2) $(p<0.05)$ and CD127 (IL-7R) $(p<0.01)$ marker expression compared with women without a concomitant autoimmune disorder. Concerning effector molecules, TNF- $\alpha$ and granzyme B production by MAIT cells was decreased.

Conclusions/interpretation Alterations in MAIT cell frequency, phenotype and function were more pronounced in adults with long-term type 1 diabetes compared with adults with recent-onset type 1 diabetes. There were several correlations between MAIT cell variables and clinical characteristics. Moreover, the presence of another autoimmune disease in women with long-term type 1 diabetes further exacerbated MAIT cell alterations. Our results suggest that MAIT cell alterations in adults with type 1 diabetes could be associated with two aspects of the disease: impaired glucose homeostasis; and autoimmunity.
\end{abstract}

Keywords Autoimmunity $\cdot$ Cytokine $\cdot$ Granzyme $\cdot$ Human $\cdot$ Innate immunity $\cdot$ MAIT cells $\cdot$ Type 1 diabetes

Agnès Lehuen

agnes.lehuen@inserm.fr

1 Institut Cochin, Inserm, CNRS, Laboratory of Excellence Inflamex,

Université de Paris, Paris, France

2 Diabetology Department, Cochin Hospital, AP-HP Centre -

Université de Paris, Paris, France 


\section{Research in context}

\section{What is already known about this subject?}

- Studies in mouse models have shown the involvement of mucosal-associated invariant T (MAIT) cells in type 1 diabetes physiopathology

- MAIT cell frequency, phenotype and function are altered in children with type 1 diabetes

- Type 1 diabetes is a heterogenous disease, notably depending on the age of individuals

\section{What is the key question?}

- What are MAIT cell characteristics in adults with type 1 diabetes?

\section{What are the new findings?}

- $\quad$ Alterations in MAIT cell phenotype and function are more pronounced in adults with long-term type 1 diabetes than in adults with recent-onset type 1 diabetes

- Some MAIT cell alterations correlated with $\mathrm{HbA}_{1 \mathrm{c}}$ level

- MAIT cell alterations are more pronounced in women with long-term type 1 diabetes who suffer from another autoimmune disease

\section{How might this impact on clinical practice in the foreseeable future?}

- More pronounced MAIT cell alterations in individuals with long-term type 1 diabetes and the link between MAIT cell alteration and impaired glucose homeostasis suggest that analysis of MAIT cells in individuals with long-term type 1 diabetes might help to monitor diabetes evolution

$\begin{array}{ll}\text { Abbreviations } \\ \text { BCL-2 } & \text { B-cell lymphoma } 2 \\ \text { DN } & \text { Double negative }\left(\mathrm{CD}^{-} \mathrm{CD}^{-}\right) \\ \text {GzB } & \text { Granzyme B } \\ \text { LADA } & \text { Latent autoimmune diabetes in adults } \\ \text { mAb } & \text { Monoclonal antibody } \\ \text { MAIT } & \text { Mucosal-associated invariant T } \\ \text { MFI } & \text { Mean fluorescence intensity } \\ \text { MR1 } & \text { MHC-related 1 protein } \\ \text { PBMC } & \text { Peripheral blood mononuclear cell } \\ \text { PCA } & \text { Principal component analysis } \\ \text { PD1 } & \text { Programmed cell death 1 } \\ \text { PMA } & \text { Phorbol myristate acetate }\end{array}$

\section{Introduction}

Type 1 diabetes results from the progressive dysfunction and destruction of insulin-secreting pancreatic beta cells [1-3]. It is a multifactorial autoimmune disease in which the innate and the adaptive immune systems are both implicated [3-6]. The resulting hyperglycaemia leads to a requirement for lifelong insulin administration and exposes affected individuals to debilitating micro- and macrovascular complications, which can be fatal [1]. Susceptibility to type 1 diabetes depends on genetic predispositions and environmental factors [1-3]. Half the cases of type 1 diabetes are declared before puberty but the disease can occur at any age [3]. Moreover, approximately $25 \%$ of individuals with type 1 diabetes also display a second autoimmune pathology, such as autoimmune thyroiditis or coeliac disease [7, 8].

Mucosal-associated invariant T (MAIT) cells are innatelike $\mathrm{T}$ lymphocytes that express a semi-invariant $\alpha \beta \mathrm{T}$ cell antigen receptor [9-11]. In humans, MAIT cells are found in several tissues and are preferentially located in blood and liver, where they represent $1-10 \%$ of T cells [12-14]. They not only recognise a limited number of ligands presented on the MHC-related 1 (MR1) protein, mainly derived from the bacteria riboflavin metabolite pathway, but also can be activated by cytokines [13, 15-19]. Activated MAIT cells produce numerous cytokines (including TNF- $\alpha$, IFN- $\gamma$, IL-4, IL-17 and IL-22) and display cytotoxic activity $[12,13,20,21]$.

Several studies have implicated MAIT cells in autoimmune diseases [22], and we and others recently reported alterations of MAIT cells in children affected by type 1 diabetes [20, 23] and their involvement in mouse models of this pathology [20, 24]. MAIT cell frequency is decreased and they present an activated, exhausted phenotype in the blood of children affected by recent-onset type 1 diabetes [20]. MAIT cells display cytotoxic activity in the pancreas of NOD mice and they can directly kill a human beta pancreatic cell line in vitro [20]. 
However, type 1 diabetes is an heterogenous disease in individuals, notably depending on age [25-29]. Beta cell destruction occurs faster in children than in adults and ketoacidosis is more frequent at onset in children than in adults [30]. Similarly, MAIT cell frequency and phenotype vary according to age $[31,32]$. Such heterogeneity of clinical and biological variables suggests therefore potential differences between children and adults in both involvement of MAIT cells and pathophysiological mechanisms of type 1 diabetes.

Here, we characterised circulating MAIT cells in adults with recent-onset type 1 diabetes and in adults with longterm type 1 diabetes compared with healthy individuals, to determine MAIT cell profiles according to diabetes evolution. We also evaluated the impact of the association of diabetes with another autoimmune disease on MAIT cells in women with long-term type 1 diabetes.

\section{Methods}

Healthy adult donors and individuals with type 1 diabetes Peripheral blood samples were collected from 55 healthy blood donors (individuals with diabetes were excluded for blood donation) by the French blood organisation (Etablissement Français du Sang) and from 47 adults with long-term type 1 diabetes (follow-up >2 years), 21 adults with recent-onset type 1 diabetes (Table 1 ) and 17 adult women suffering from long-term type 1 diabetes and another autoimmune disease (Table 2) from Cochin hospital, Paris. Women in the last group were affected by Graves' disease (4/17), Hashimoto's thyroiditis (9/17), autoimmune thyroiditis $(3 / 17)$ or juvenile polyarthritis (1/17). Type 1 diabetes was diagnosed according to current medical recommendations based on clinical manifestations and biological variables. All individuals with type 1 diabetes presented autoantibodies against at least one of the three antigens GAD, islet antigen 2 (IA2) or zinc transporter 8 (ZnT8) at diagnosis. Individuals with latent autoimmune diabetes in adults (LADA) (age at onset more than 35 years and insulin requirement less than 1 year) or ketosis-prone diabetes were excluded from the study, as well as those with ongoing infectious/inflammatory diseases. Samples were mainly analysed before the COVID19 outbreak in France (February 2020). Otherwise, blood was taken only from individuals without clinical symptoms of COVID-19 or positive PCR for SARS-CoV-2. Patients with ongoing antibiotic or immunomodulatory treatments were also excluded from this study. Participants with long-term type 1 diabetes were compared with healthy donors of similar age, BMI and sex ratio. The selected participants with longterm type 1 diabetes did not present any renal, cardiac or severe ophthalmological complications.

Our study was approved by the Ethics Committee (comité de protection des personnes (CPP) Ile-de-France) (N Eudra CT/ID-RCB: 2014-A00517-40) and all participants provided written informed consent before blood donation.

Peripheral blood mononuclear cell preparation and flow cytometry analysis After isolation from fresh blood samples using Ficoll-Paque (Leucosep) tubes, peripheral blood mononuclear cells (PBMCs) were first labelled in PBS containing $5 \%$ FCS and $0.1 \%$ sodium azide with the following antihuman monoclonal antibodies (mAbs): anti-CD3 (OKT3, cat. no. 317329, RRID:AB_11219196), CD4 (OKT4, cat. no. 317440, RRID:AB_2562912), V $\alpha 7.2$ (3C10, cat. no. 351715, RRID:AB_2562534), CD161 (HP-3G10, cat. no. 339915, RRID:AB_11142679), CCR6 (G034E3, cat. no. 353410, RRID:AB_10913815), CD56 (HCD56, cat. no. 318340, RRID:Ā__2561944), CD69 (FN50, cat. no. 310933, RRID:AB_2561783), CD27 (O323, cat. no. 302835, RRID:AB_2561382) from BioLegend (San Diego, CA, USA); anti-CD8 (SK1, cat. no. 557834 , RRID:AB_396892), Programmed cell death 1 (PD1) (MIH4, cat. no. 557860, RRID:AB_2159176), CD25 (MA251, cat. no. 557741, RRID:AB_396847) from BD Biosciences (San Jose, CA, USA); and anti-CD127 (R34.34,
Table 1 Clinical and biological characteristics of individuals with type 1 diabetes and healthy donors

\begin{tabular}{llll}
\hline Characteristic & Healthy donors & RO T1D & LT T1D \\
\hline$n$ & 55 & 21 & 47 \\
Age, years & $37.6 \pm 13.5(22.2-67.3)$ & $30.0 \pm 12.9(16.9-69.1)$ & $38.1 \pm 14.6(19.8-74.1)$ \\
BMI, $\mathrm{kg} / \mathrm{m}^{2}$ & $22.9 \pm 3.4(18.0-32.6)$ & $21.4 \pm 2.9(16.6-27.7)$ & $24.0 \pm 4.4(17.4-39.6)$ \\
Sex ratio M/F & $25 / 30$ & $11 / 10$ & $20 / 27$ \\
$\mathrm{~T} 1 \mathrm{D}$ duration & - & $6 \pm 4$ days $(2-14$ days $)$ & $18.2 \pm 13.2$ years $(2-53$ years $)$ \\
$\mathrm{HbA}_{1 \mathrm{c}}, \mathrm{mmol} / \mathrm{mol}$ & - & $97.1 \pm 29.0(41.0-168.9)$ & $63.0 \pm 18.2(32.2-125.1)$ \\
$\mathrm{HbA}_{1 \mathrm{c}}, \%$ & - & $11.0 \pm 2.7(5.9-17.6)$ & $7.9 \pm 1.7(5.1-13.6)$ \\
Daily insulin dose, $\mathrm{U} / \mathrm{kg}$ & - & $0.6 \pm 0.3(0.2-1.4)$ & $0.6 \pm 0.2(0.2-1.4)$ \\
\hline
\end{tabular}

Data are presented as mean $\pm \mathrm{SD}$ (range) unless stated otherwise

The adults with recent-onset or long-term type 1 diabetes do not have an associated autoimmune disease F, female; LT, long-term; M, male; RO, recent-onset; T1D, type 1 diabetes 
Table 2 Clinical and biological characteristics of healthy women, women with long-term type 1 diabetes or with long-term type 1 diabetes and another autoimmune disease

\begin{tabular}{llll}
\hline Characteristic & Healthy donors & LT T1D & LT T1D and another AID \\
\hline$n$ & 30 & 27 & 17 \\
Age, years & $35.8 \pm 13.0(22.2-67.3)$ & $39.4 \pm 15.5(19.8-71.5)$ & $47.8 \pm 15.7(23.5-75.7)$ \\
BMI, kg/m & $22.3 \pm 3.0(18.0-29.5)$ & $24.5 \pm 5.1(17.4-39.6)$ & $23.7 \pm 2.9(19-28.3)$ \\
T1D duration, years & - & $18.6 \pm 15.3(2-53)$ & $25.1 \pm 14.5(2-54)$ \\
$\mathrm{HbA}_{1 \mathrm{c}}, \mathrm{mmol} / \mathrm{mol}$ & - & $65.5 \pm 17.9(39.9-125.1)$ & $64.7 \pm 10.7(48.6-79.2)$ \\
$\mathrm{HbA}_{1 \mathrm{c}}, \%$ & - & $8.1 \pm 1.6(5.8-13.6)$ & $8.1 \pm 1.0(6.6-9.4)$ \\
\hline
\end{tabular}

Data are presented as mean \pm SD (range) unless stated otherwise

AID, autoimmune disease; LT, long-term; T1D, type 1 diabetes cat. no. A64617, RRID:AB 2833010) from Beckman (Marseille, France).

For B-cell lymphoma 2 (BCL-2) and Ki67 detection after surface staining, PBMCs were resuspended in fixationpermeabilisation buffer (eBiosciences, Thermo Fisher Scientific, Carlsbad, CA, USA) and incubated protected from light at $4^{\circ} \mathrm{C}$ with $\mathrm{mAbs}$ anti-Ki67 (B56, cat. no. 556027 , RRID:AB 2266296) from BD Biosciences and anti-BCL-2 (clone 100, cat. no. 658704, RRID:AB 2563152) from BioLegend. PBMCs were then washed with perm wash buffer (eBiosciences).

For detection of cytokines and granzyme B (GzB), PBMCs were activated with phorbol myristate acetate (PMA) (25 ng/ml; Sigma-Aldrich, MO, USA) and ionomycin $(1 \mu \mathrm{g} / \mathrm{ml}$; Sigma-Aldrich) in the presence of brefeldin A $\left(10 \mu \mathrm{g} / \mathrm{ml}\right.$; Sigma-Aldrich) for $6 \mathrm{~h}$ at $37^{\circ} \mathrm{C}$ in RPMI medium supplemented with $10 \%$ FCS. After surface staining, PBMCs were fixed and permeabilised using the Cytofix/Cytoperm kit (BD Biosciences), then washed and stained, protected from light at $4{ }^{\circ} \mathrm{C}$, with the following mAbs: anti-IFN- $\gamma$ (4S B3, cat. no. 506504, RRID:AB_315437), IL-2 (MQ1-17H12, cat. no. 500306, RRID:AB_315093), IL-17 (BL168, cat. no. 512327, RRID:AB_11219603) and TNF- $\alpha$ (Mab11, cat. no. 502937, RRID:AB_2561355) from BioLegend; and anti-IL-4 (8D4-8, cat. no. 560672, RRID:AB_1727547), IL-10 (JES3-19F1, cat. no.554707, RRID:AB_398582) and GzB (GB11, cat. no. 563388, RRID:AB_2738174) from BD Biosciences.

According to the number of PBMCs isolated from each participant or control individual, surface staining was always realised, and when possible intracellular staining of GzB and cytokines after PMA-ionomycin stimulation or of BCL-2 and Ki67 without stimulation. Data acquisition was performed using a BD Biosciences LSR-Fortessa cytometer or a FACSAria III cytometer and results were analysed with FlowJo analysis software V10.1 (Tree Star).

Statistical and bioinformatic analyses Multivariable analysis pie charts were built using Excel 2016 (https://www. microsoft.com, Microsoft, Redmond, WA, USA) and FlowJo analysis software V10.1 (https://www.flowjo.com, Becton, Dickinson and Company, San Jose, CA, USA, Tree Star). Principal component analysis (PCA) and correlograms were realised as previously described [33]. Briefly, PCA was processed with FactoMineR package and visualised with Factoextra package. Correlograms were produced with the rcorr function from Hmisc and RcmdrMisc packages to compute pairwise-complete matrices of Spearman's correlations along with the asymptotic $p$ values and visualised with the Corrplot and ggplot2 packages. Datasets were filtered for missing values, and only complete observations were used for Spearman's correlations. Dplyr library including the tidyverse package was used to sort and filter datasets. Statistical analyses were performed with GraphPad Prism V8.3.0 (https:// www.graphpad.com, Graphpad Software, San Diego, CA,

Fig. 1 Frequency and phenotype alterations of circulating MAIT cells in adults with type 1 diabetes. PBMCs $\left(5 \times 10^{6}\right)$ were collected from healthy donors $(n=55)$ and from adults with recent-onset $(n=21)$ or long-term $(n=47)$ type 1 diabetes and were analysed by flow cytometry. $(\mathbf{a}-\mathbf{c})$ Circulating MAIT (a), MAIT CD8 ${ }^{+} \mathrm{CD} 4^{-}$(b) and MAIT CD8 ${ }^{-} \mathrm{CD} 4^{-}$ (DN) (c) cell frequencies among $\mathrm{CD}^{+}$cells. $(\mathbf{d}-\mathbf{i})$ Frequencies of $\mathrm{CCR}^{+}(\mathbf{d}), \mathrm{CD}_{6} 6^{+}(\mathbf{e}), \mathrm{CD} 9^{+}(\mathbf{f}), \mathrm{CD}^{+} 5^{+}(\mathbf{g}), \mathrm{PD}^{+}(\mathbf{h})$ or $\mathrm{CD} 27^{+}(\mathbf{i})$ MAIT cells among total MAIT cells. $(\mathbf{j}-\mathbf{0})$ Correlations between frequency of circulating MAIT cells and frequency of $\mathrm{CD} 25^{+}(\mathbf{j}-\mathbf{l})$ or $\mathrm{PD}^{+}(\mathbf{m}-\mathbf{o})$ MAIT cells in healthy donors and participants with recentonset or long-term type 1 diabetes. (p-r) Frequencies of $\mathrm{Ki}^{+}(\mathbf{p})$, $\mathrm{CD}_{127^{+}}(\mathbf{q})$ or BCL-2 ${ }^{+}(\mathbf{r})$ MAIT cells among total MAIT cells in healthy donors ( $n=42,55,42$ for $\mathbf{p}, \mathbf{q}, \mathbf{r}$, respectively) and participants with recent-onset ( $n=7,21,7$, respectively) or long-term ( $n=33,47,33$, respectively) type 1 diabetes. (s-u) Correlations between frequency of circulating MAIT cells and frequency of CD $127^{+}$MAIT cells in healthy donors $(n=55)(\mathbf{s})$ and participants with recent-onset $(n=21)$ (t) or long-term $(n=47)(\mathbf{u})$ type 1 diabetes. $(\mathbf{v}-\mathbf{x})$ Correlograms of circulating MAIT cell frequency and marker expression in healthy donors $(n=42-55)(\mathbf{v})$ and participants with recent-onset $(n=7-21)(\mathbf{w})$ or long-term $(n=33-47)(\mathbf{x})$ type 1 diabetes. Only significant Spearman's correlation coefficients are represented, by colour intensity and square size. Each symbol represents a single individual (a-u) and small horizontal lines indicate the median with the IQR $(\mathbf{a}-\mathbf{i}, \mathbf{p}-\mathbf{r}) . * p<0.05$, $*^{*} p<0.01$ and $* * * p<0.001(\mathbf{a}-\mathbf{i}, \mathbf{p}-\mathbf{r}) ;{ }^{\S} p=0.062(\mathbf{a}) ;{ }^{\dagger} p=0.051(\mathbf{g})$; ${ }^{\ddagger} p=0.052$ (i) (non-parametric two-tailed Mann-Whitney test [a-i, $\left.\mathbf{p}-\mathbf{r}\right]$ or Spearman's correlation test $[\mathbf{j}-\mathbf{0}, \mathbf{s}-\mathbf{x}])$. HD, healthy donors; LT, longterm; RO, recent-onset; T1D, type 1 diabetes 

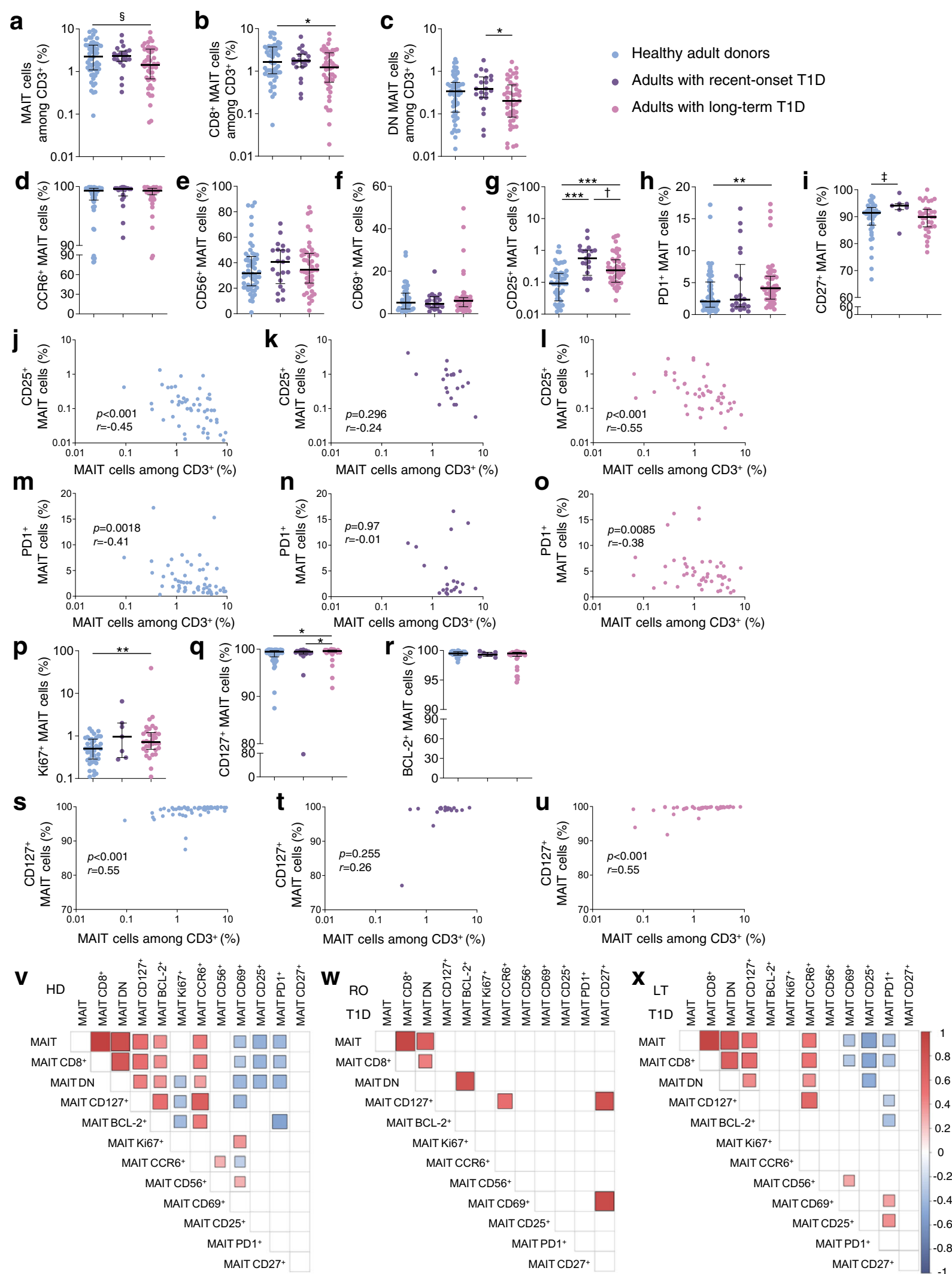

$\mathbf{X}_{\text {LT }} \quad$ 总 z
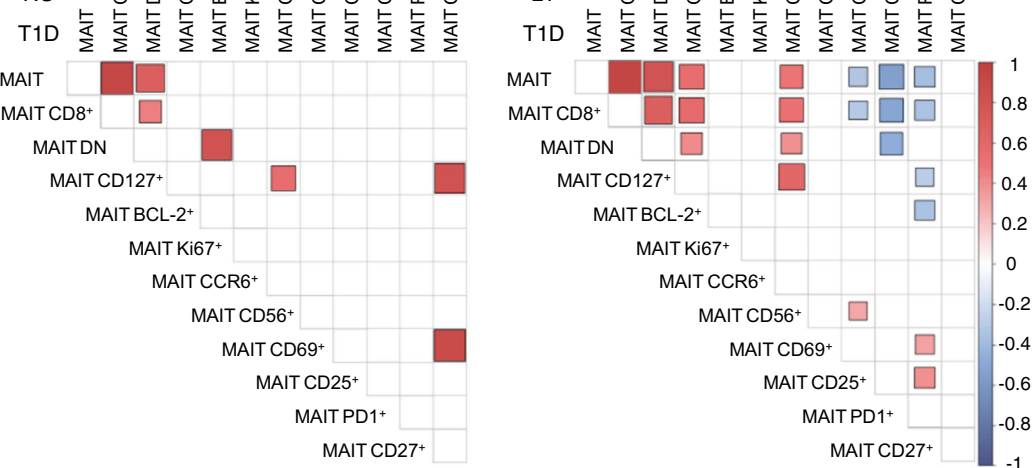
USA) or R software V4.0 (https://www.r-project.org/) with RStudio V1.2.5 [34]. Datasets were all tested for normal distribution using the Shapiro-Wilk normality test. All datasets were compared using either non-parametric twotailed Wilcoxon-Mann-Whitney test or non-parametric Spearman's correlation test with exact $p$ values, as appropriate. Differences with a $p<0.05$ were considered significant.

\section{Results}

Circulating MAIT cell phenotype is altered in adults with type 1 diabetes We first characterised MAIT cells in fresh peripheral blood samples from adults with longterm type 1 diabetes and from adults with recent-onset type 1 diabetes as compared with healthy adult blood donors. Clinical and biological variables of participants with type 1 diabetes and the healthy donors are reported in Table 1. MAIT cells were identified by flow cytometry as CD161 ${ }^{\text {high }} \mathrm{V} \alpha 7.2^{+} \mathrm{T}$ cells (ESM Fig. 1a), identical to Ag-loaded MR1 tetramer identification in blood [33, 35, 36]. In human blood, MAIT cells are divided in two main subsets, $\mathrm{CD}^{+}$and $\mathrm{CD}^{-} \mathrm{CD}^{-}$(double negative, DN), usually accounting for $80 \%$ and $15 \%$ of total MAIT cells, respectively (ESM Fig. 1a) [13, 33]. In the participants with long-term type 1 diabetes, $\mathrm{CD}^{+}$MAIT cell frequency among all $\mathrm{T}$ cells was slightly reduced, as compared with healthy adult donors (Fig. 1a, b). There was also a reduction in DN MAIT cell frequency in participants with long-term compared with recent-onset diabetes (Fig. 1c). This suggests altered homeostasis of MAIT cells in individuals with long-term diabetes.

We next analysed the surface phenotype of circulating MAIT cells in the participants with diabetes and the control donors (Fig. 1d-i, ESM Fig. 1b). Frequencies of MAIT cells expressing the tissue-recruitment molecule CCR6 or the adhesion/activation molecule CD56 were similar between the three groups (Fig. 1d, e), suggesting no differences in tissue migration capability. We also quantified frequencies of MAIT cells for expression of markers of early and late activation (CD69 and CD25) as well as markers of sustained activation/exhaustion (PD1). We did not observe any differences in $\mathrm{CD}^{+} 9^{+}$ MAIT cell frequencies between control donors and participants with recent-onset or long-term type 1 diabetes (Fig. 1f). However, the frequency of $\mathrm{CD} 25^{+}$MAIT cells was significantly increased in individuals with recent-onset type 1 diabetes and, to a lesser extent, in those with long-term type 1 diabetes (Fig. $1 \mathrm{~g}$ ), when compared with the frequency in healthy donors. Moreover, PD $1^{+}$MAIT cell frequency was significantly higher in individuals with long-term type 1 diabetes compared with healthy control individuals (Fig. 1h). Expression of the activation marker
Fig. 2 Functional alterations of circulating MAIT cells are correlated in adults with type 1 diabetes. (a-g) Flow cytometry analysis of IL-2 ${ }^{+}$(a), $\mathrm{IFN}-\gamma^{+}(\mathbf{b}), \mathrm{TNF}-\alpha^{+}(\mathbf{c}), \mathrm{IL}-17^{+}(\mathbf{d}), \mathrm{IL}_{-} 4^{+}(\mathbf{e}), \mathrm{GzB}^{+}(\mathbf{f})$ and IL-10 $0^{+}(\mathbf{g})$ MAIT cells among total MAIT cells in healthy donors $(n=42)$, and adults with recent-onset $(n=10)$ or long-term $(n=37)$ type 1 diabetes. $(\mathbf{h}-\mathbf{j})$ Pie charts showing MAIT cells producing Th1 cytokines, IL-17 and/or GzB, in healthy donors (h), adults with recent-onset type 1 diabetes (i) or longterm type 1 diabetes $(\mathbf{j})$. (k) Bar plot representative of cumulative Th1 cytokines, IL-17 and GzB, production by MAIT cells, classified as producing none (negative), one (monofunction), two (bifunction) or more than two (polyfunction) of these factors. (I) Circular bar plot represents negative $\log _{e} p$ values of the proportion differences between healthy donors $(n=42)$ and adults with recent-onset $(n=10)$ or long-term $(n=37)$ type 1 diabetes. Red dashed circles represent $p$ values. Each symbol represents a single individual and small horizontal lines indicate median with IQR $(\mathbf{a}-\mathbf{g}) . * p<0.05, * * p<0.01$ and $* * * p<0.001$ (nonparametric two-tailed Mann-Whitney test $[\mathbf{a}-\mathbf{g}, \mathbf{l}])$. HD, healthy donors; LT, long-term; RO, recent-onset; T1D, type 1 diabetes

CD27 on MAIT cells was increased in participants with recent-onset type 1 diabetes compared with healthy donors $(p=0.052)$ (Fig. 1i). Interestingly, both $\mathrm{CD} 25^{+}$ and $\mathrm{PD}^{+}$MAIT cell frequencies negatively correlated with MAIT cell frequency in both healthy donors and participants with long-term type 1 diabetes but not in those with recent-onset type 1 diabetes (Fig. $1 j-0$ ). This points toward an increased activation status of MAIT cells in individuals with recent-onset type 1 diabetes and also exhaustion of these cells in individuals with long-term type 1 diabetes.

We also analysed expression of markers associated with cell proliferation (Ki67) and survival (CD127 and BCL-2) (Fig. 1p-r and ESM Fig. 1b). Ki67 ${ }^{+}$MAIT cell and CD $127^{+}$ MAIT cell frequencies were significantly higher in individuals with long-term type 1 diabetes compared with healthy control individuals (Fig. 1p, q). No differences in BCL-2 $2^{+}$MAIT cell frequency were found between the three groups (Fig. 1r). CD $127^{+}$MAIT cell frequency positively correlated with blood MAIT cell frequency in participants with long-term type 1 diabetes (Fig. 1u). A similar correlation was observed in healthy control donors but not in participants with recentonset type 1 diabetes (Fig. 1s, t). Alterations in Ki67 and CD127 expression thus further support specific alteration of MAIT cell homeostasis in individuals with long-term diabetes.

We next performed correlograms with all these MAIT cell variables (Fig. $1 \mathrm{v}-\mathrm{x}$ ). In healthy adult donors, we identified a first cluster of positive correlations between blood MAIT cell frequencies (total MAIT, CD8 ${ }^{+}$MAIT, DN MAIT) and frequencies of MAIT cells expressing tissue residency (CCR6) and survival markers (CD127, BCL-2) (Fig. 1v). Conversely, there were several negative correlations between blood MAIT cell frequencies and frequencies of MAIT cells expressing proliferation (Ki67) and activation markers (CD69, CD25, PD1). In individuals with recent-onset type 1 diabetes, correlations 

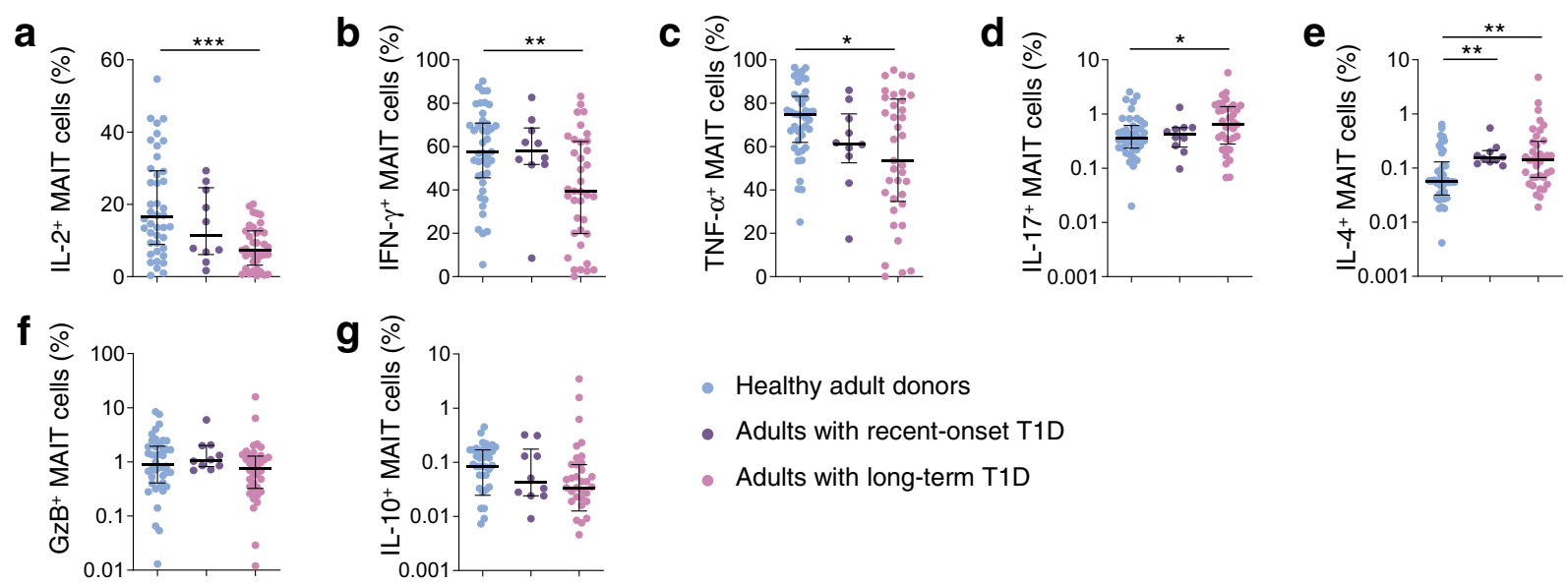

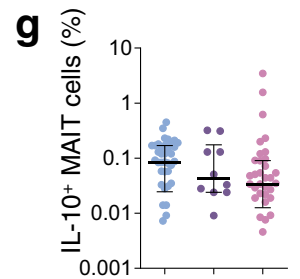

h Healthy adult donors

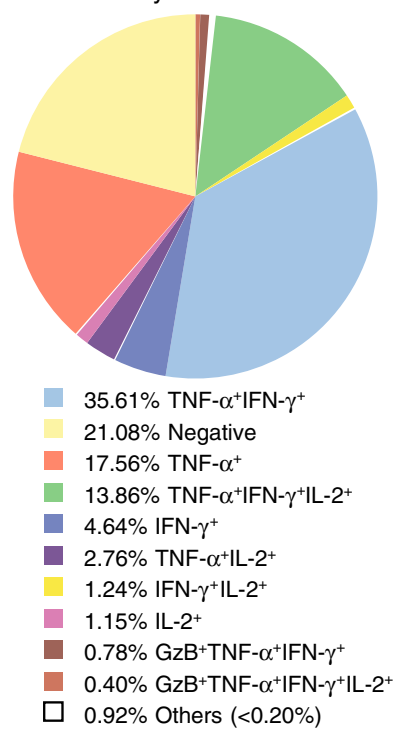

i Adults with RO T1D

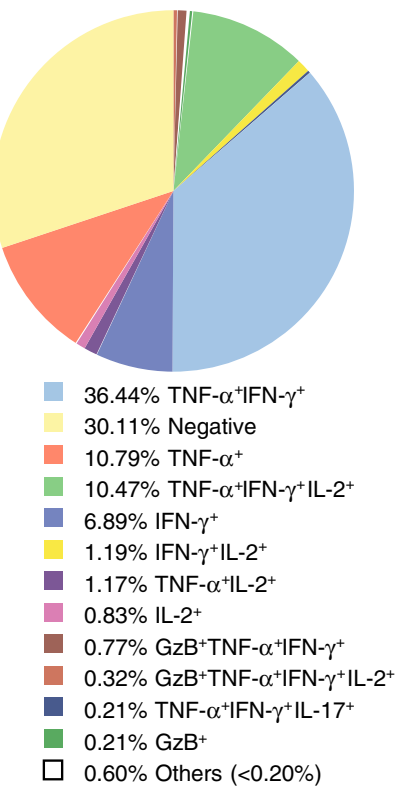

- Healthy adult donors

- Adults with recent-onset T1D

- Adults with long-term T1D

\section{k}

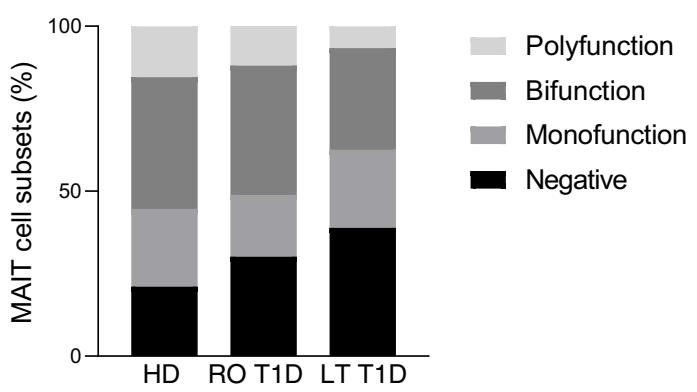

j Adults with LT T1D

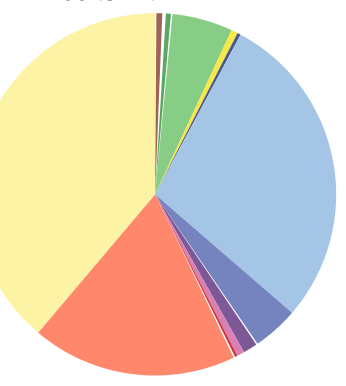

$38.85 \%$ Negative

$28.52 \%$ TNF- $\alpha^{+}$IFN- $\gamma^{+}$

$18.34 \%$ TNF- $\alpha^{+}$

$5.42 \%$ TNF- $\alpha^{+}$IFN- $\gamma^{+}$IL-2 ${ }^{+}$

$4.09 \%$ IFN- $\gamma^{+}$

$1.27 \%$ TNF- $\alpha^{+} \mathrm{IL}-2^{+}$

$0.68 \% \mathrm{IL}-2^{+}$

$0.58 \%$ IFN- $\gamma^{+} \mathrm{IL}-2^{+}$

$0.53 \% \mathrm{GzB}+\mathrm{TNF}-\alpha^{+} \mathrm{IFN}-\gamma^{+}$

$0.44 \% \mathrm{GzB}^{+}$

- $0.30 \%$ TNF- $\alpha^{+}$IFN- $\gamma^{+}$IL- $17^{+}$

$0.21 \%$ TNF- $\alpha^{+} \mathrm{IL}-17^{+}$

$\square \quad 0.77 \%$ Others (<0.20\%)

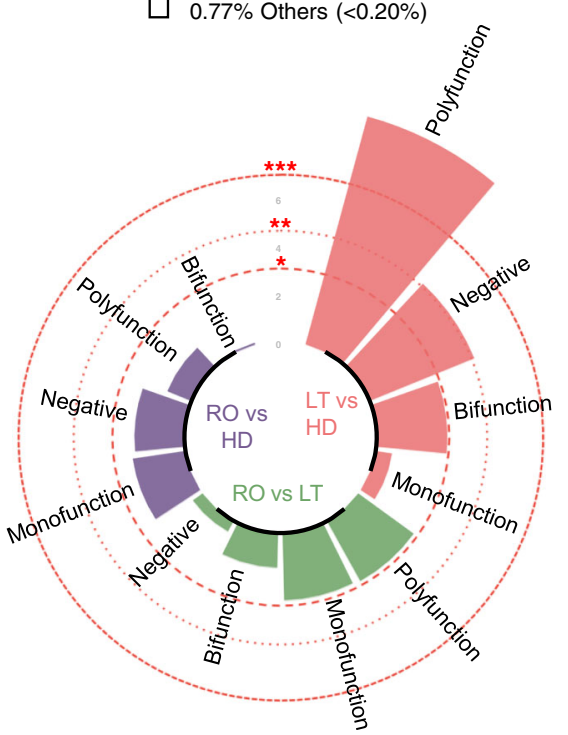


between blood MAIT cell frequencies and frequencies of MAIT cells expressing proliferation, activation and survival markers were lost when compared with healthy control donors (Fig. 1w). However, these clusters were conserved in adults with long-term type 1 diabetes compared with healthy adult donors, with the notable exception of the negative correlations with $\mathrm{Ki}^{+} 7^{+}$MAIT cell frequency (Fig. 1x). Altogether, our results reveal different MAIT cell alterations in adults with recentonset or long-term type 1 diabetes.

Cytokine and GzB production in MAIT cells from adults with type 1 diabetes We next investigated MAIT cell function by analysing production of Th1 (IL-2, IFN- $\gamma$ and TNF- $\alpha$ ) cytokines, IL-17, GzB, IL-4 and IL-10 after PMA-ionomycin stimulation (Fig. 2a-g). In healthy control individuals MAIT cells produced high levels of Th1 cytokines whereas levels were significantly decreased in individuals with long-term type 1 diabetes (Fig. 2a-c and ESM Fig. 2). IL-17 and IL-4 production by MAIT cells slightly increased in individuals with long-term type 1 diabetes compared with healthy control individuals (Fig. 2d, e and ESM Fig. 2). In participants with recent-onset type 1 diabetes, only IL-4 production was increased. GzB and IL-10 levels in MAIT cells were similar between the three groups (Fig. 2f, g). Of note, cytokine and GzB staining on MAIT cells showed no difference in mean fluorescence intensity (MFI) between the three groups (ESM Fig. 3a).

We further analysed the ability of MAIT cells to produce Th1 cytokines, IL-17 and GzB, by multivariable analysis (Fig. $2 \mathrm{~h}-\mathrm{j}$ ). Data showed the functional heterogeneity of MAIT cell subsets in the three groups of participants and, among those producing cytokines, the main subsets produced one, two or three Th1 cytokines. In healthy donors and participants with type 1 diabetes, cytokine-producing MAIT cell subsets were classified as producing none, one (monofunctional), two (bifunctional) or more than two (polyfunctional) different cytokines or GzB to display their functional heterogeneity. Between healthy donors and individuals with type 1 diabetes, there was a progressive increase in the frequency of MAIT cells producing none of the effector molecules analysed (negative subset), from healthy donors to individuals with recent-onset and finally long-term type 1 diabetes (Fig. $2 \mathrm{k}$ ). Conversely, the frequency of polyfunctional and bifunctional subsets decreased in patients with recentonset and long-term type 1 diabetes compared with healthy controls (Fig. 2k). This subset distribution differed significantly between healthy donors and individuals with long-term type 1 diabetes (respectively, median $19.30 \%$ and $34.40 \%$ of MAIT cells for 'negative' MAIT cells, $p<0.01$ and median $13.14 \%$ vs $4.8 \%$ of MAIT cells
Fig. 3 MAIT cell alterations are distinct between adults with recent-onset and long-term type 1 diabetes. (a, b) PCA of healthy donors $(n=42)$ and adults with recent-onset $(n=10)$ and long-term $(n=37)$ type 1 diabetes, using MAIT cell phenotype and function markers as variables. Each small point represents a single individual and the mean value for each group is represented by a larger symbol (a); arrows represent the contribution made by each quantitative variable and concentration ellipses indicate 95\% CIs (b). (c-e) Correlograms of circulating MAIT cell phenotype and function in healthy donors $(n=33-55)(\mathbf{c})$, adults with recent-onset type 1 diabetes $(n=5-21)$ (d) and adults with long-term type 1 diabetes $(n=29-47)$ (e). Only significant Spearman's correlation coefficients are represented by colour intensity and square size. (f-n) Correlations between TNF- $\alpha^{+}$and IFN- $\gamma^{+}$MAIT cell frequencies $(\mathbf{f}-\mathbf{h})$, between IL$17^{+}$and IL-2 $2^{+}$MAIT cell frequencies (i-k), and between IL- $17^{+}$and IL$4^{+}$MAIT cell frequencies $(\mathbf{I}-\mathbf{n})$ in healthy donors $(n=42$; blue circles), adults with recent-onset type 1 diabetes ( $n=10$; purple circles) or longterm type 1 diabetes ( $n=37$; pink circles). Each point represents a single individual (f-n). HD, healthy donors; LT, long-term; PC, Principal component; RO, recent-onset; T1D, type 1 diabetes

for polyfunctional MAIT cells, $p<0.001$ ) (Fig. 21 and ESM Fig. 3b).

We next performed a PCA to show the distribution of the three groups of individuals according to MAIT cell frequency, phenotype and functional variables. Participants with long-term type 1 diabetes were more distinctly isolated from healthy control individuals than those with recent-onset type 1 diabetes (Fig. 3a). Decreased Th1 cytokines and increased CD69, PD1 and IL-4 expression were the major contributors to the segregation of adults with long-term type 1 diabetes from heathy donors (Fig. 3b). We next performed correlograms of MAIT cell frequency, phenotype and function in healthy control individuals and participants with type 1 diabetes. A cluster of positive correlations between the three Th1 cytokines was observed in healthy donors and adults with long-term type 1 diabetes, whereas only TNF- $\alpha$ and IFN- $\gamma$ MAIT cell production correlated with each other in adults with recent-onset type 1 diabetes (Fig. $3 \mathrm{c}-\mathrm{h}$ and ESM Fig. 3c). Interestingly, MAIT cell IL-17 production positively correlated with MAIT cell TNF- $\alpha$ production in healthy donors and also with other Th1 cytokine production (IFN- $\gamma$ and IL-2) in individuals with long-term type 1 diabetes. In these individuals, MAIT cell IL-17 production also positively correlated with MAIT cell IL-4 production (Fig. 3c, e, i-n). Correlations between MAIT cell effector molecule production and MAIT cell frequency and phenotype were globally different between the three groups. Altogether, there are distinct MAIT cell phenotypic and functional alterations between individuals with recent-onset and long-term type 1 diabetes.

MAIT cell alterations correlate with diabetes-associated clinical variables We next investigated whether MAIT cell frequency, phenotype and functions were associated with clinical 
a

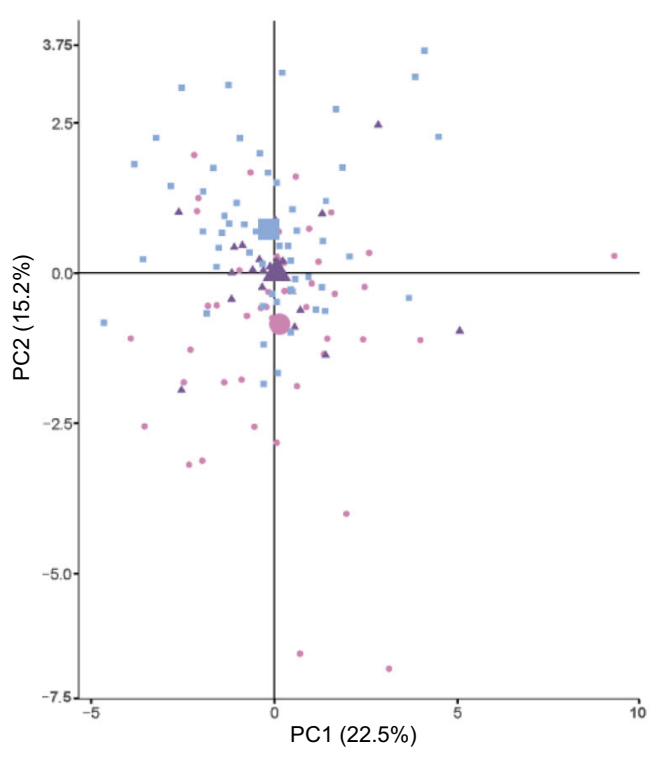

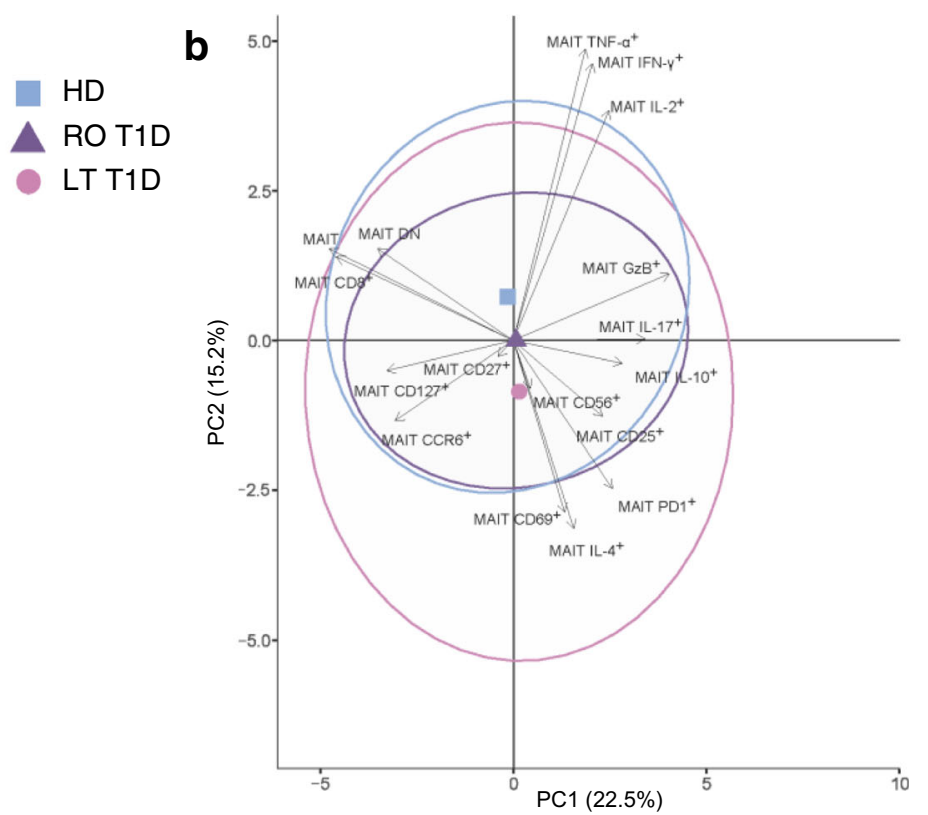

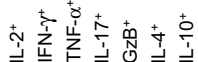

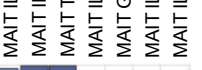

MAIT

MAIT CD8 ${ }^{+}$ MAIT DN MAIT CD $127^{+}$ MAIT CCR6 ${ }^{+}$

MAIT CD56 ${ }^{+}$ MAIT CD69+ MAIT CD25+

MAIT PD ${ }^{+}$ MAIT CD $27^{+}$

MAIT IL-2+ MAIT IFN- $\gamma^{+}$ MAIT TNF- $\alpha^{+}$ MAIT IL-17+

MAIT GzB ${ }^{+}$

MAIT IL- $4^{+}$

MAIT IL-10+

MAIT IL-4+

MAIT IL-10+

f

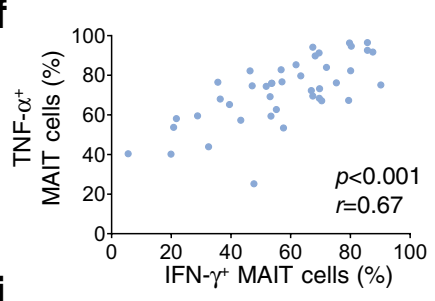

i

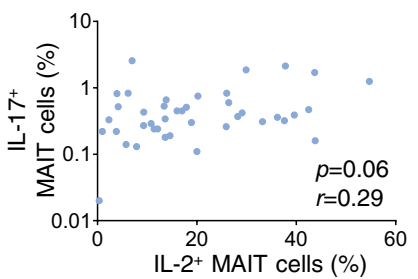

I

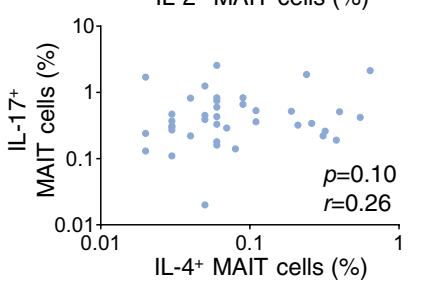

g

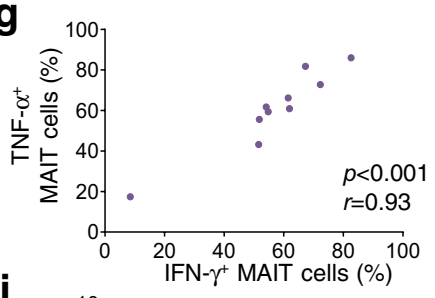

j

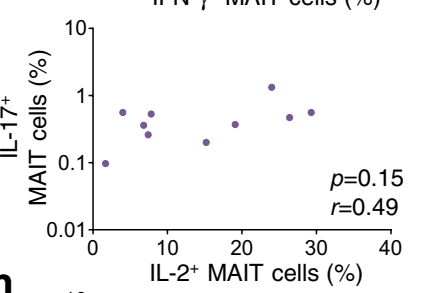

m

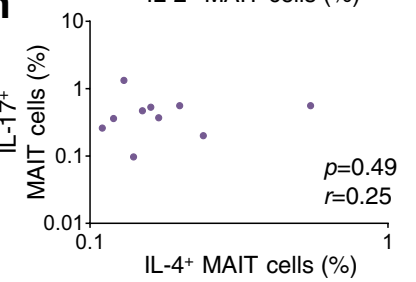

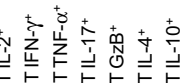

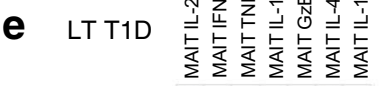

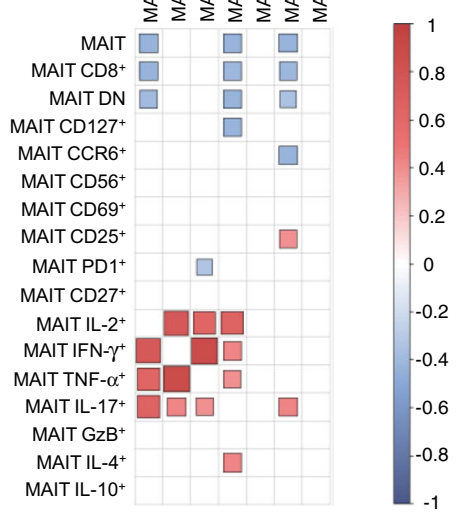

h
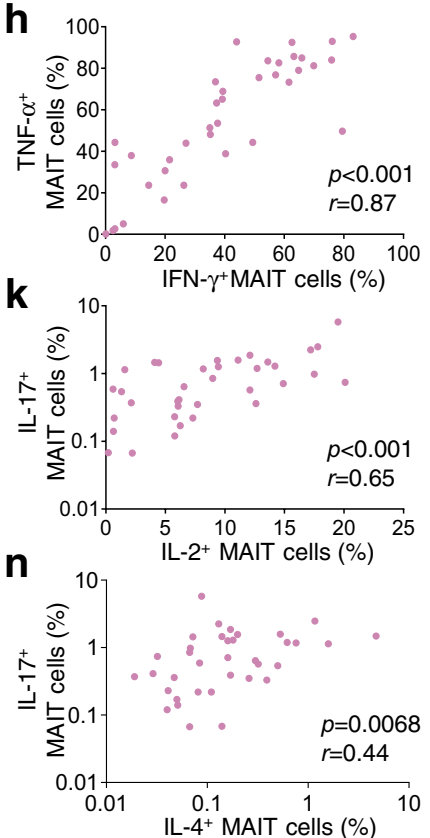
variables in adults with type 1 diabetes. In participants with recent-onset type 1 diabetes, correlations were observed between $\mathrm{HbA}_{1 \mathrm{c}}$ level and MAIT cell variables such as $\mathrm{CD}_{6}{ }^{+}, \mathrm{Ki}^{+}, \mathrm{GzB}^{+}$and $\mathrm{IL}-4^{+}$MAIT cell frequencies (Fig. $4 \mathrm{a}-\mathrm{f})$. MFI of IL-4 staining also correlated with $\mathrm{HbA}_{1 \mathrm{c}}$ in these individuals. In adults with long-term type 1 diabetes, despite the low variability of $\mathrm{HbA}_{1 \mathrm{c}}$ level, $\mathrm{PD}^{+}$MAIT cell frequency positively correlated with $\mathrm{HbA}_{1 \mathrm{c}}$ level (Fig. $4 \mathrm{a}, \mathrm{g}$ ). In these individuals, $\mathrm{CD} 69^{+}$MAIT cell frequency positively correlated with daily insulin dose (Fig. 4a, g-i). This underlines several associations between MAIT cell alterations and impaired glucose homeostasis in individuals with recent-onset type 1 diabetes and, to a lesser extent, in those with long-term type 1 diabetes.

\section{Exacerbated MAIT cell alterations in women with long-term} type 1 diabetes and another autoimmune disease We next assessed the impact of another autoimmune disease in association with type 1 diabetes on MAIT cell alterations. Since associated autoimmune diseases mainly occurred in women with diabetes, we compared MAIT cells from women with long-term type 1 diabetes plus another autoimmune disease with those from the other female participants, both healthy and with long-term type 1 diabetes (Table 2). Frequencies of total MAIT cells, $\mathrm{CD}^{+}$MAIT cells and DN MAIT cells were significantly decreased in women with long-term type 1 diabetes and another autoimmune disease compared with healthy women (Fig. 5ac). In these women, CD69 expression on MAIT cells was significantly increased compared with healthy women and women with long-term type 1 diabetes (Fig. 5d-h). Expression of survival markers CD127 and BCL-2 by MAIT cells was decreased in women with long-term type 1 diabetes and another autoimmune disease compared with other groups (Fig. 5i-1). Expression of these markers, as well as the frequency of total MAIT cells, negatively correlated with CD69 expression (Fig. 5m-o). Several correlations between CD27 and other MAIT cell variables, such as a positive correlation with MAIT cell frequency and negative correlations with CD69 and PD1 expression by MAIT cells, were only observed in the women with long-term type 1 diabetes and another autoimmune disease (Fig. 5p-u). Altogether, this highlights several modifications of MAIT cells in women with long-term type 1 diabetes and another autoimmune disease that differed from those occurring in either of the other cohorts of women.

Analysis of IL-2, IFN- $\gamma$ and TNF- $\alpha$ showed that the proportion of MAIT cells producing these cytokines was reduced in women with long-term type 1 diabetes compared with healthy donors regardless of autoimmune disease status (Fig. 6a-c). Among the variables studied, the MFI of MAIT cells after staining was lower for
TNF- $\alpha$ and GzB in women with long-term type 1 diabetes and another autoimmune disease compared with women with long-term type 1 diabetes (Fig. 6d, g and ESM Fig. 4a). Of note these two variables correlated with each other in all three groups of women analysed (Fig. 6j-1 and ESM Fig. 4b). As previously shown with individuals with longterm type 1 diabetes, women from this group also displayed an elevated frequency of the negative cytokine/GzB-producing MAIT cell subset compared with healthy women (Fig. 6m and ESM Fig. 4c). MAIT cells from women with long-term type 1 diabetes and women with long-term type 1 diabetes and another autoimmune disease presented a similar profile. In both groups, MAIT cells were less multifunctional compared with healthy female donors, with decreased polyfunctional and bifunctional MAIT cell frequencies (Fig. 6n). PCA revealed a better segregation from healthy female donors of women with long-term diabetes and another autoimmune disease than women with long-term diabetes (Fig. 6o, p). MAIT cell (total, $\mathrm{CD}^{+}, \mathrm{DN}$ ) frequencies and CD69 expression contributed to segregation between women with long-term diabetes with or without another autoimmune disease. Therefore, we revealed common and distinct MAIT cell alterations between both groups of women with long-term diabetes.

\section{Discussion}

The present study shows that MAIT cells are altered in adults with type 1 diabetes. These alterations in phenotype and function are more pronounced in adults with long-term type 1 diabetes compared with those with recent-onset type 1 diabetes. Several MAIT cell alterations positively correlated to glucose homeostasis impairment. Moreover, the presence of another autoimmune disease in women with long-term type 1 diabetes exacerbated MAIT cell alterations (ESM Table 1).

In individuals with recent-onset type 1 diabetes, we demonstrated that MAIT cells harboured an activated profile (CD25 and CD27 upregulation) without major alterations in cell homeostasis and function. In contrast, in individuals with long-term type 1 diabetes, as well as CD25, MAIT cells also displayed upregulation of the exhaustion marker PD1 and molecules involved in proliferation (Ki67) and survival (CD127). $\mathrm{CD}^{+}$and $\mathrm{DN}$ MAIT cell frequencies were decreased in these individuals and this increased proliferation and survivability might be a mechanism to compensate for their lower frequencies. MAIT cell frequency negatively correlated with exhausted MAIT cell frequency and positively with $\mathrm{CD} 127^{+}$MAIT cell frequency. Such correlations were not observed in individuals with recent-onset type 1 diabetes, 
a

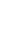

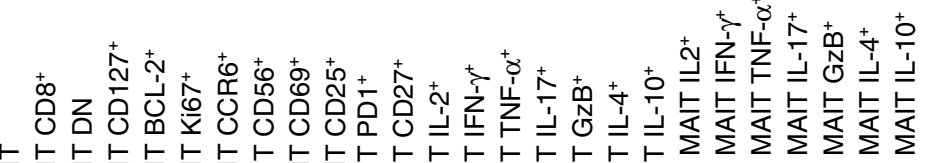

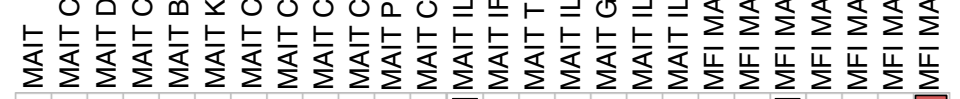

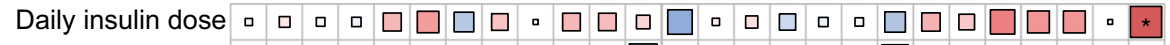

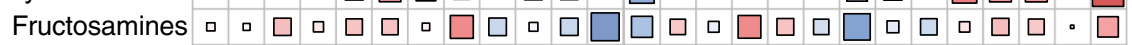

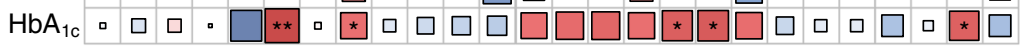

\section{Recent-onset T1D}

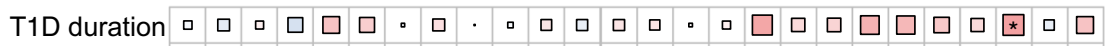

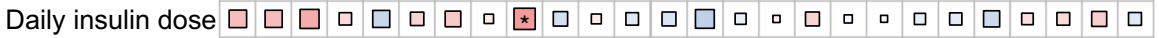
Fructosamines •

\section{Long-term T1D}

$\mathrm{HbA}_{1 \mathrm{c}}$ ・
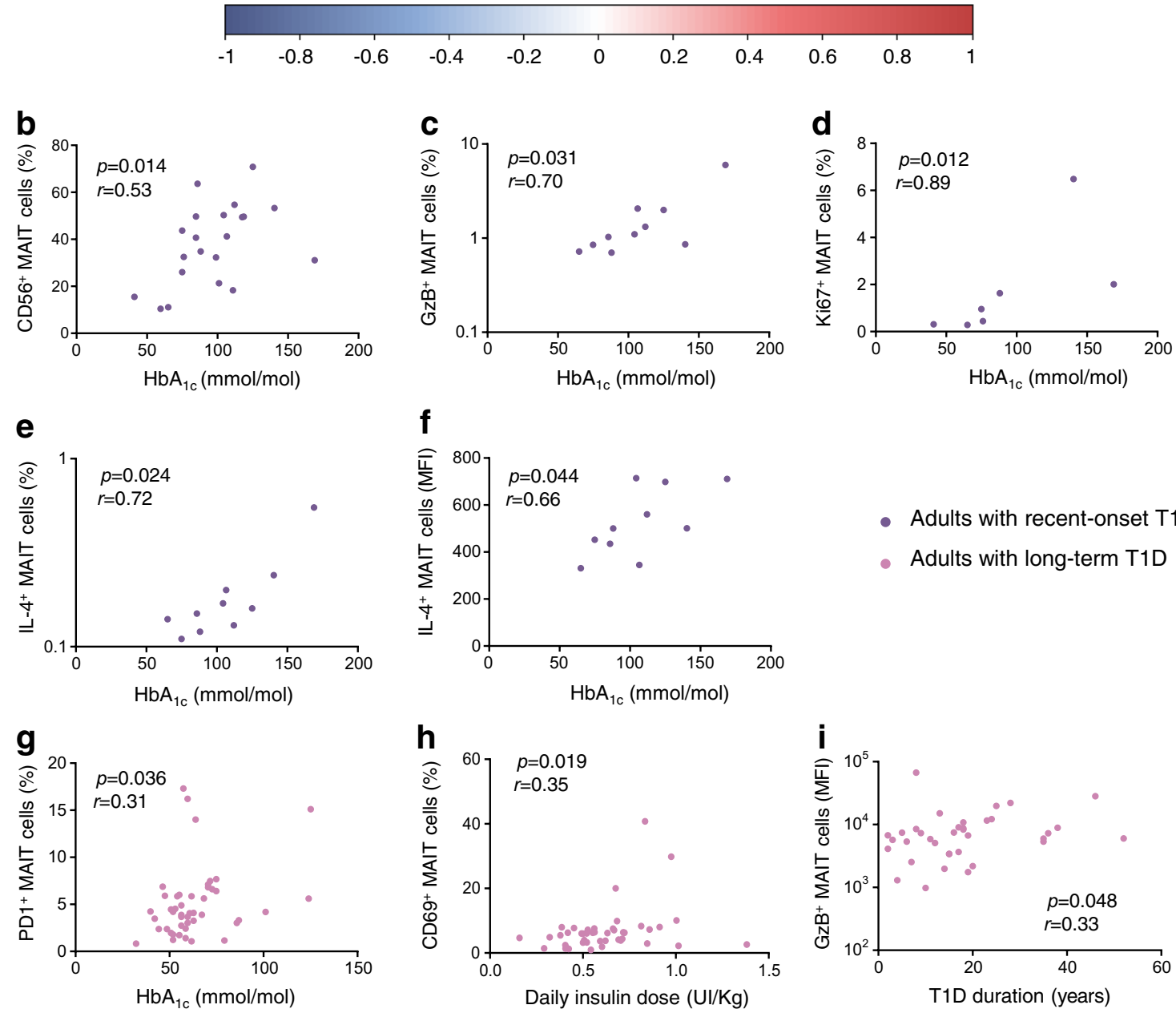

- Adults with recent-onset T1D
- Adults with long-term T1D

Fig. 4 MAIT cell alterations correlated with glycaemic variables in adults with type 1 diabetes. (a) Correlograms of circulating MAIT cell frequency, phenotype and function previously analysed and clinical variables (daily insulin dose, fructosamine level, $\mathrm{HbA}_{1 \mathrm{c}}$ level and type 1 diabetes duration) in adults with recent-onset $(n=5-21)$ or long-term $(n=24-47)$ type 1 diabetes. Spearman's correlation coefficients are represented by colour intensity and square size. Asymptotic $p$ values are displayed. All correlations are shown, with asterisks denoting those that are significant.

(b-f) Correlations between $\mathrm{CD}^{+} 6^{+}(\mathbf{b}), \mathrm{GzB}^{+}(\mathbf{c}), \mathrm{Ki}^{+} 7^{+}(\mathbf{d})$ and $\mathrm{IL}-4^{+}$ (frequency, $\mathbf{e}$ and MFI of positive cells, $\mathbf{f}$ ) MAIT cells with $\mathrm{HbA}_{1 \mathrm{c}}$ level in adults with recent-onset type 1 diabetes. $(\mathbf{g}-\mathbf{i})$ Correlations between $\mathrm{PD} 1^{+}$ MAIT cells and $\mathrm{HbA}_{1 \mathrm{c}}$ level $(\mathbf{g})$, between $\mathrm{CD} 69^{+}$MAIT cells and daily insulin dose (h), and between MFI of $\mathrm{GzB}^{+}$MAIT cells and type 1 diabetes duration (i) in adults with long-term type 1 diabetes. Each symbol represents a single individual $(\mathbf{b}-\mathbf{i}) .{ }^{*} p<0.05$ and $* * p<0.01$ (non-parametric Spearman's correlation test $[\mathbf{a}-\mathbf{i}]$ ). T1D, type 1 diabetes 

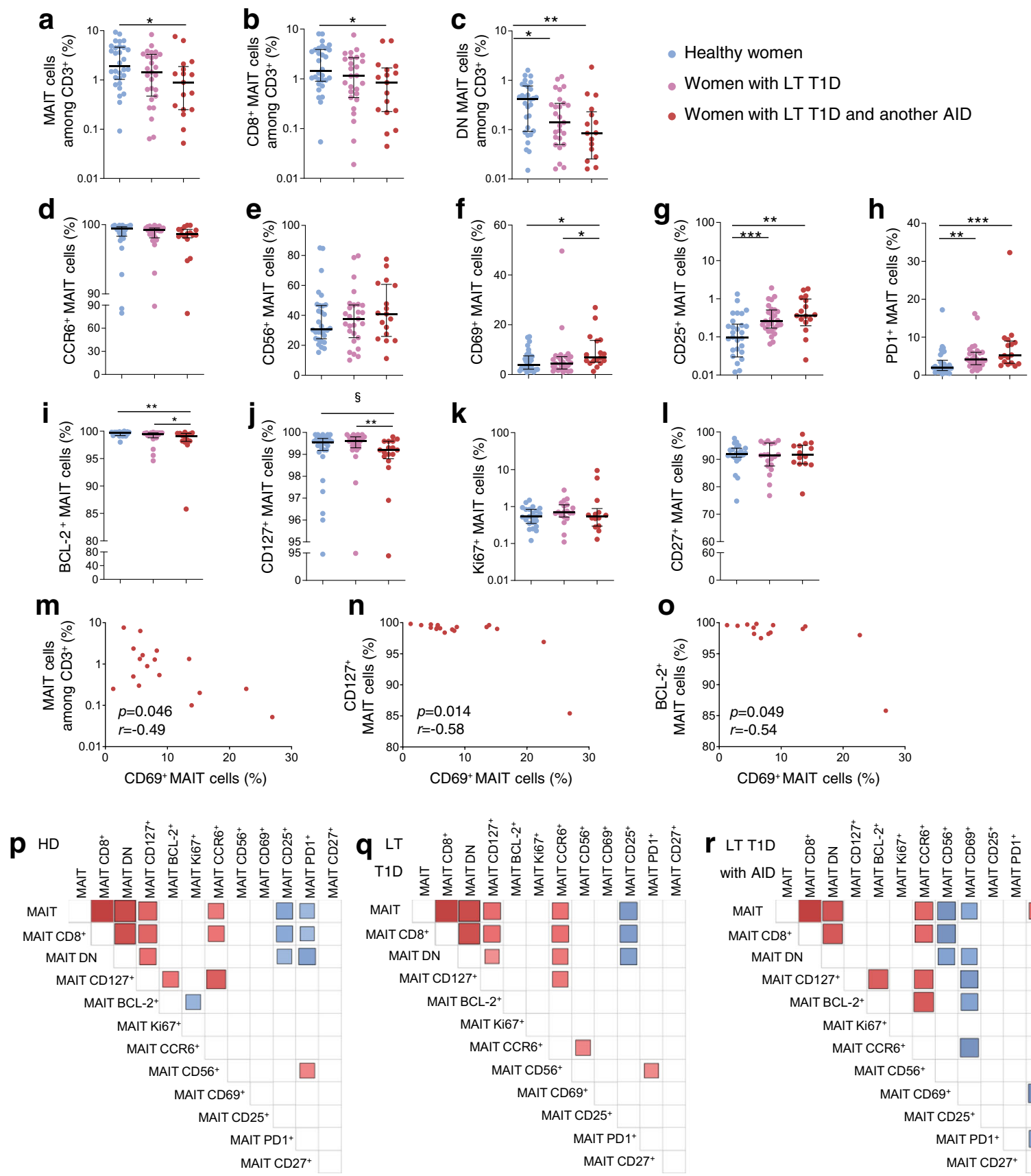

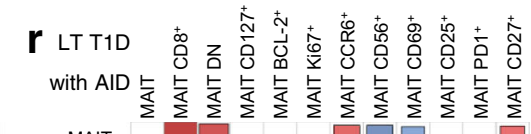
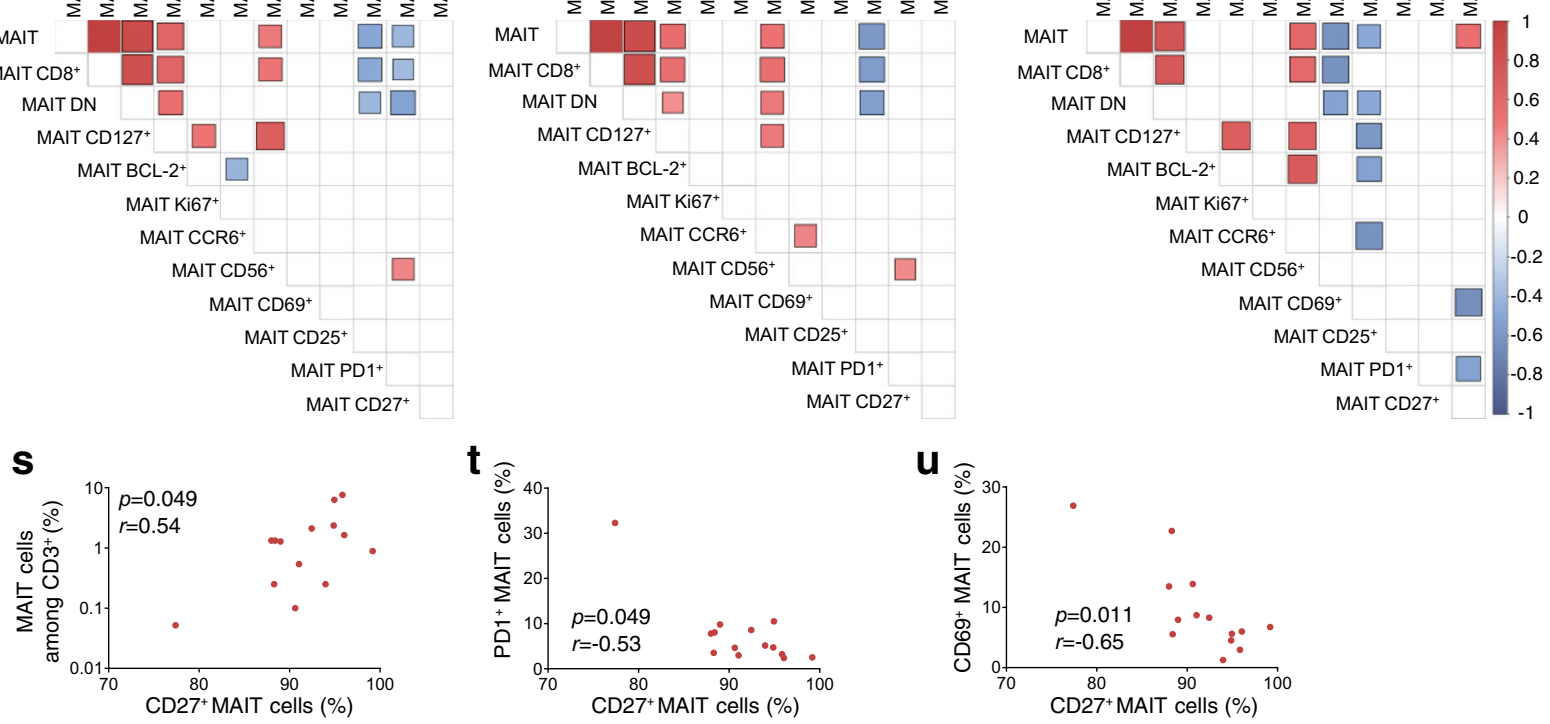
4 Fig. 5 Frequency and phenotype alterations of circulating MAIT cells in women with type 1 diabetes and another autoimmune disease. PBMCs $\left(5 \times 10^{6}\right)$ were collected from healthy female donors $(n=30)$, women with long-term type 1 diabetes $(n=27)$ or women with long-term type 1 diabetes and another autoimmune disease $(n=17)$ and analysed by flow cytometry. (a-c) Circulating MAIT (a), MAIT CD8 ${ }^{+} \mathrm{CD} 4^{-}$(b) and MAIT CD8 ${ }^{-} \mathrm{CD}^{-}{ }^{-}(\mathrm{DN})(\mathbf{c})$ cell frequencies among $\mathrm{CD}^{+}$cells. $(\mathbf{d}-\mathbf{l})$ Frequencies of $\mathrm{CCR}^{+}(\mathbf{d}), \mathrm{CD}^{+} 6^{+}(\mathbf{e}), \mathrm{CD} 9^{+}(\mathbf{f}), \mathrm{CD}^{2} 5^{+}(\mathbf{g}), \mathrm{PD}^{+}$ (h), BCL-2 ${ }^{+}(\mathbf{i}), \mathrm{CD} 127^{+}(\mathbf{j}), \mathrm{Ki}^{+}(\mathbf{k})$ and $\mathrm{CD} 27^{+}$(l) MAIT cells among total MAIT cells. (m-o) Correlations between circulating MAIT $(\mathbf{m}), \mathrm{CD} 127^{+}$MAIT (n) or BCL-2 $2^{+}$MAIT (o) cell frequencies and CD69 ${ }^{+}$ MAIT cell frequency in women with long-term type 1 diabetes and another autoimmune disease $(n=17)$. ( $\mathbf{p}-\mathbf{r})$ Correlograms of circulating MAIT cell frequency and phenotype markers in healthy female donors $(n=23-30)(\mathbf{p})$, women with long-term type 1 diabetes $(n=19-27)(\mathbf{q})$ or long-term type 1 diabetes with another autoimmune disease $(n=14-17)$ $(\mathbf{r})$. Only significant Spearman's correlation coefficients are represented by colour intensity and square size. (s-u) Correlations between circulating MAIT $(\mathbf{s}), \mathrm{PD}^{+}(\mathbf{t})$ or $\mathrm{CD}^{+} 9^{+}(\mathbf{u})$ MAIT cell frequencies and $\mathrm{CD} 27^{+}$MAIT cell frequency in women with long-term type 1 diabetes and another autoimmune disease $(n=17)$. Each symbol represents a single individual $(\mathbf{a}-\mathbf{0}, \mathbf{s}-\mathbf{u})$ and small horizontal lines indicate the median with the IQR $(\mathbf{a}-\mathbf{I}) . * p<0.05, * * p<0.01$, $* * * p<0.001(\mathbf{a}-\mathbf{0}, \mathbf{s}-\mathbf{u})$ and ${ }^{\S} p=0.0533(\mathbf{j})$ (non-parametric two-tailed Mann-Whitney test $[\mathbf{a}-\mathbf{l}]$ or Spearman's correlation test $[\mathbf{m}-\mathbf{u}]$ ). AID, autoimmune disease; HD, healthy donors; LT, long-term; RO, recentonset; T1D, type 1 diabetes

suggesting that MAIT cell profiles may differ as the disease evolves. Whether these differences depend on disease history or may predict future complications remains to be investigated.

In our study, we addressed whether these heterogenous MAIT cell alterations were related to diabetes-associated clinical variables. In individuals with recent-onset type 1 diabetes, $\mathrm{Ki}_{67}{ }^{+}, \mathrm{CD} 6^{+}, \mathrm{IL}_{-} 4^{+}$and $\mathrm{GzB}^{+}$MAIT cell frequency positively correlated with blood $\mathrm{HbA}_{1 \mathrm{c}}$ level. Production of $\mathrm{IL}_{-} 4^{+}$and $\mathrm{GzB}^{+}$is elevated in MAIT cells from children with recent-onset type 1 diabetes, although $\mathrm{GzB}^{+}$MAIT cell frequency inversely correlates with blood $\mathrm{HbA}_{1 \mathrm{c}}$ [20]. As type 1 diabetes is less aggressive in adults [30], these correlations may reflect a slowly rising blood glucose level before disease diagnosis, linked with progressive alterations of MAIT cells [20]. In contrast, in our study participants with long-term type 1 diabetes, $\mathrm{HbA}_{1 \mathrm{c}}$ levels positively correlated only with $\mathrm{PD}^{+}$MAIT cell frequency. This could result from a negative impact of the increased blood glucose level on MAIT cell frequency and function as already described in obesity and type 2 diabetes [37, 38]. Alternatively, higher PD1 expression by MAIT cells could reflect a chronic activation status due to increased general inflammation levels in individuals with less-well-controlled diabetes [36-38]. Of note, our present cohort was composed of individuals without complications and who had globally well-controlled blood glucose levels, so it would be of interest to study eventual differences in the status of MAIT cells in uncontrolled type 1 diabetes.

In agreement with this increased PD1 expression, the production of Th1 cytokines was decreased in MAIT cells from individuals with long-term type 1 diabetes. Circulating MAIT cells in humans mainly produce Th1 cytokines, such as IL-2 (18\%), IFN- $\gamma(60 \%)$ and TNF- $\alpha$ (75\%) [13]. Decreased Th1 cytokine production by MAIT cells was associated with increased frequency of MAIT cells that synthesised none of the cytokines analysed nor GzB, and a loss of polyfunctional MAIT cells. Even though there was a global shift of Th1 to IL-17 and IL4 cytokines by MAIT cells in individuals with long-term type 1 diabetes compared with healthy donors, the production of all these cytokines strongly and positively correlated with each other. These data suggest that among the individuals with long-term type 1 diabetes, some harboured an elevated frequency of MAIT cells producing all cytokines (Th1, IL-17 and IL-4) whereas others possessed MAIT cells with a general low level of cytokine production. Moreover, multivariable analysis showed the preferential production of IL-17 in association with TNF- $\alpha$ and IFN- $\gamma$ at the cellular level. This is of importance as conventional Th1/Th17 double-producer T cells are highly pathogenic in the context of autoimmune diseases and type 1 diabetes [39-41]. Monitoring MAIT cell cytokine production may therefore help to estimate future complications. Moreover, the phenotype of MAIT cells can be significantly influenced by other immune cell populations such as monocytes and inflammatory cytokines [13, 14, 33, 42]. Similar immune cell crosstalk has been well established in type 1 diabetes $[4,43]$ and it would be interesting to link these alterations to other differences in immune cells and cytokines between individuals with recent-onset and long-term type 1 diabetes.

Type 1 diabetes is often associated with other autoimmune diseases, particularly in women [44]. The major MAIT cell alterations in women with long-term type 1 diabetes and autoimmune disease concerned MAIT cell homeostasis. Compared with women affected by long-term type 1 diabetes only, MAIT cell frequency and survivability $\left(\mathrm{BCL}-2^{+}\right.$and $\mathrm{CD} 127^{+}$frequency) were significantly decreased. CD69 ${ }^{+}$ MAIT cell frequency was increased and negatively correlated with MAIT cell frequency, BCL-2 $2^{+}$MAIT cell and CD127 MAIT cell frequencies. Even though CD69 is mainly known as an early activation marker, it is also expressed on T cells under chronic activation [45]. MAIT cells might therefore be under further sustained activation in individuals with diabetes and another autoimmune disease, although confirming this hypothesis would require comparison with individuals affected by an autoimmune disease without type 1 diabetes. Of note, corrections for multiple comparisons adjusting for the total number of statistical tests were not performed in the present 


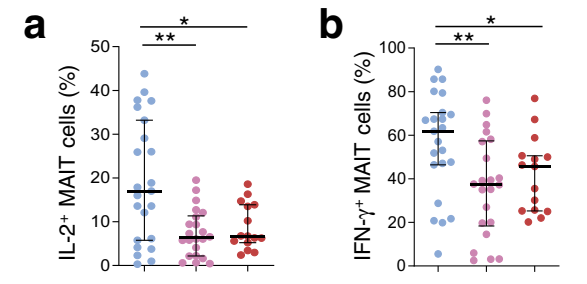

e

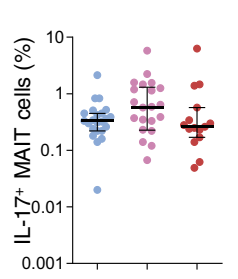

j

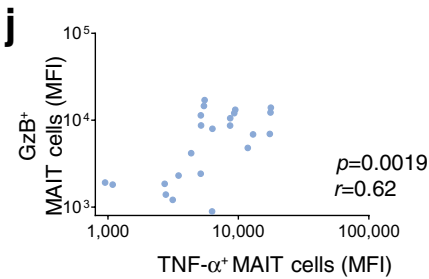

m

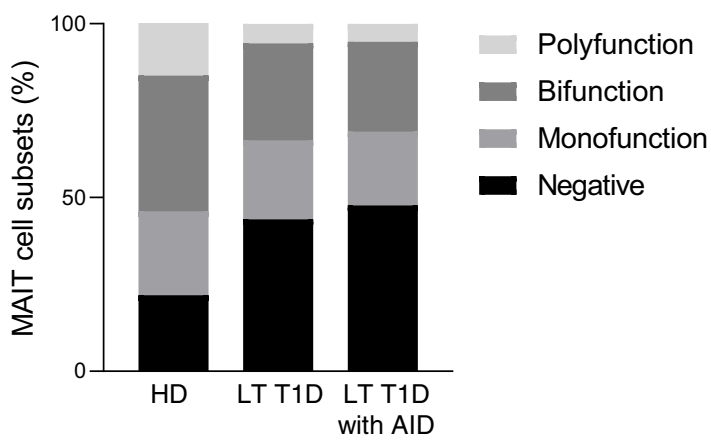

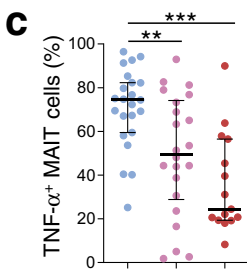

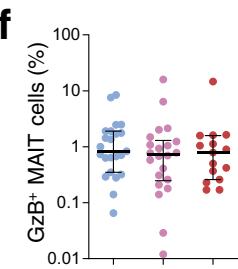

$\mathrm{g}$

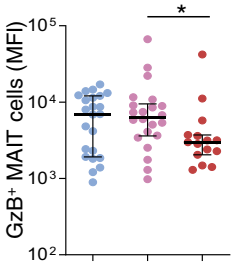

k

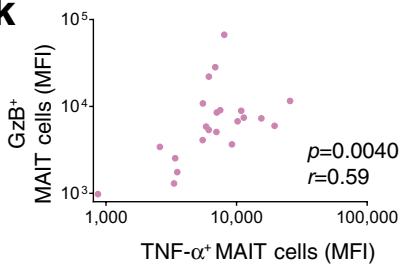

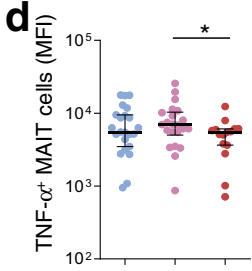

- Healthy women

- Women with LT T1D

- Women with LT T1D

and another AID

h
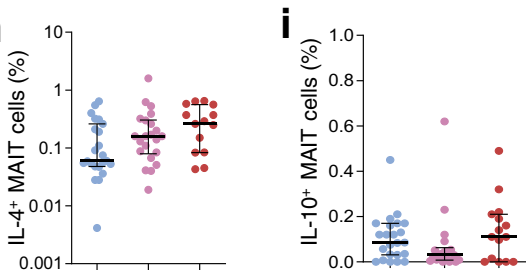

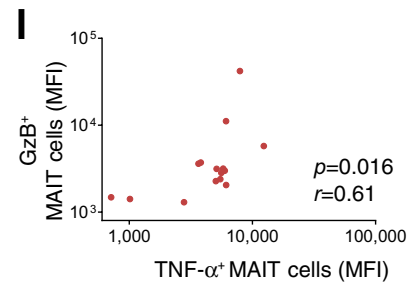

n

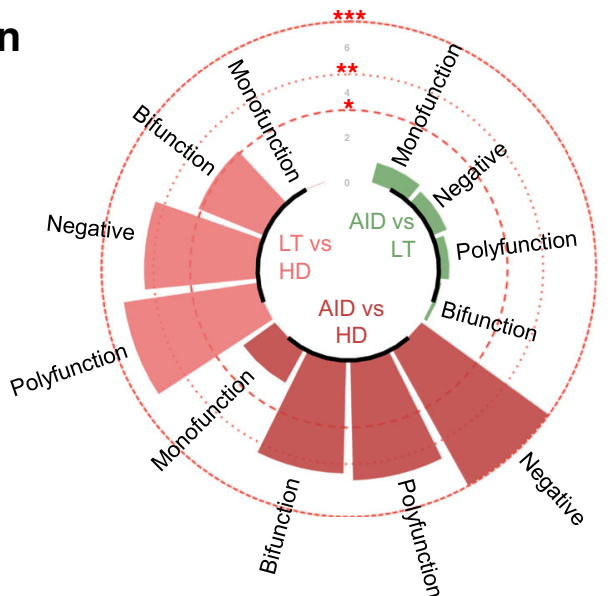

0

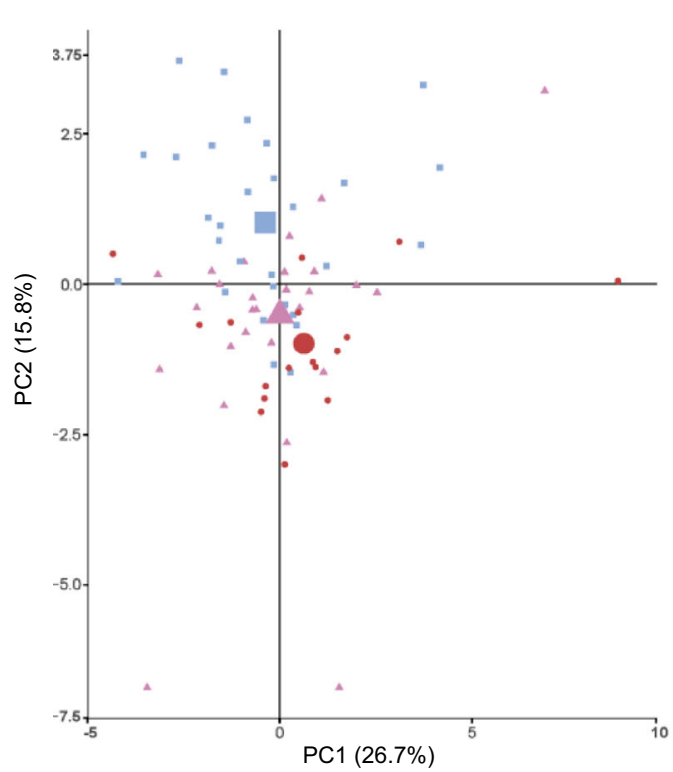

$H D$

LT T1D

LT T1D with AID

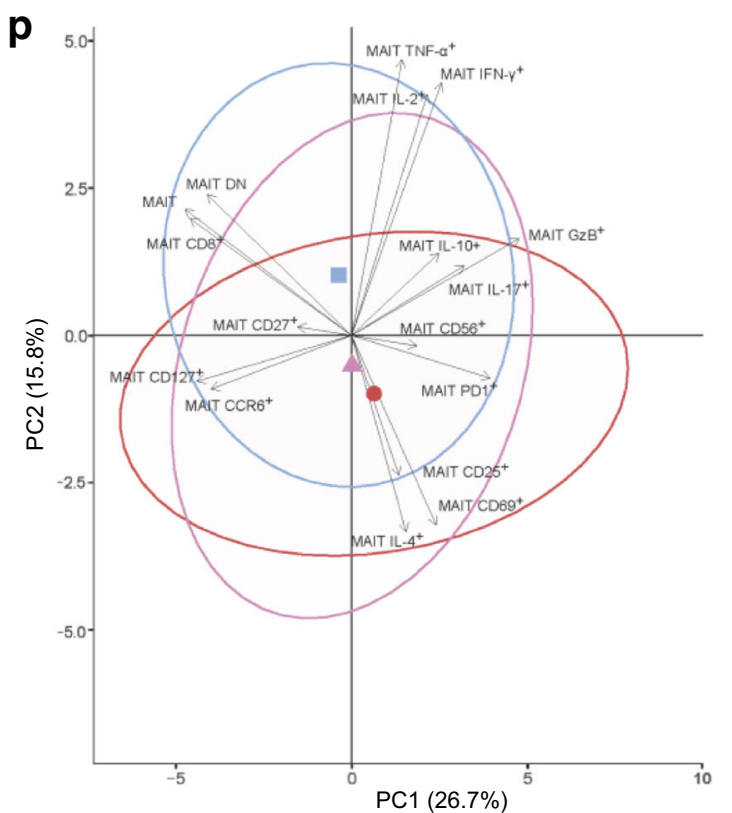


Fig. 6 Functional alterations of circulating MAIT cells in women with type 1 diabetes and another autoimmune disease. (a-i) Flow cytometry analysis of IL- $2^{+}(\mathbf{a}), \mathrm{IFN}-\gamma^{+}(\mathbf{b}), \mathrm{TNF}-\alpha^{+}(\mathbf{c}), \mathrm{IL}-17^{+}(\mathbf{e}), \mathrm{GzB}^{+}(\mathbf{f}), \mathrm{IL}-4^{+}$ (h) or IL- $10^{+}$(i) MAIT cell frequency and MFI of TNF- $\alpha^{+}$(d) or $\mathrm{GzB}^{+}$ (g). MAIT cell staining in healthy women donors $(n=23)$, women with long-term type 1 diabetes $(n=22)$ and women with long-term type 1 diabetes with another autoimmune disease $(n=15)$. (j-l) Correlations between $\mathrm{GzB}^{+}$MAIT cell MFI and TNF- $\alpha^{+}$MAIT cell MFI in healthy female donors $(n=23)(\mathbf{j})$, women with long-term type 1 diabetes $(n=22)$ (k) and women with long-term type 1 diabetes and another autoimmune disease $(n=15)$ (I). ( $\mathbf{m})$ Bar plot representative of cumulative production of Th1 cytokines, IL-17 and GzB, by MAIT cells, classified as producing none (negative), one (monofunction), two (bifunction) or more than two (polyfunction) of these factors. (n) Circular bar plot represents negative $\log _{e} p$ values of the proportion differences between healthy female donors $(n=23)$, women with long-term type 1 diabetes $(n=22)$ and women with long-term type 1 diabetes and another autoimmune disease $(n=15)$. Red dashed circles represent $p$ values. $(\mathbf{o}, \mathbf{p})$ PCA of healthy female donors $(n=23)$, women with long-term type 1 diabetes $(n=22)$ and women with long-term type 1 diabetes and another autoimmune disease $(n=15)$, using MAIT cell phenotype and function markers as variables. Each small point represents a single individual and the mean value for each group is represented by a larger symbol (o); arrows represent the contribution made by each quantitative variable and concentration ellipses indicate 95\% CIs (p). Each symbol represents a single individual $(\mathbf{a}-\mathbf{-}, \mathbf{0})$ and small horizontal lines indicate the median with the IQR $(\mathbf{a}-\mathbf{i}) . * p<0.05$, $* * p<0.01$ and $* * * p<0.001$ (non-parametric two-tailed Mann-Whitney test $[\mathbf{a}-\mathbf{i}, \mathbf{n}]$ or Spearman's correlation test $[\mathbf{j}-\mathbf{I}])$. AID, autoimmune disease; HD, healthy donors; LT, long-term; PC, Principal component; RO, recent-onset; T1D, type 1 diabetes

study since the analyses were planned before they were conducted.

Our study nonetheless reinforces the potential of MAIT cells as a biomarker of disease progression, now in adults suffering from type 1 diabetes. Our results suggest that MAIT cell alterations in adults with type 1 diabetes are linked with two aspects of the disease, one associated with autoimmunity and another with impaired glucose homeostasis.

Supplementary Information The online version of this article contains peer-reviewed but unedited supplementary material. (https://doi.org/10. 1007/s00125-021-05527-y)

Acknowledgements We thank all the patients and the medical staff of Cochin Hospital, particularly A. Solal (Diabetology Department, Cochin Hospital, AP-HP Centre, France) and the Cybio facilities of the Cochin Institute and S. Luce (Institut Cochin, Paris, France) for helping to recruit adults with recent-onset type 1 diabetes. We are grateful to U. Rogner and A. Toubal (Institut Cochin, Paris, France) for critical reading of the manuscript. The graphical abstract was designed using illustrations from Servier Medical Art, licensed under the Creative Commons Attribution 3.0 Unported License.

Data availability Corrplot datasets are available at https://doi.org/10. 6084/m9.figshare.c.5328767. All used R scripts are available at https:// github.com/MatthieuRouland/Nel-et-al. The datasets generated during and/or analysed during the current study are available from the corresponding author upon reasonable request.

Funding This study was supported by grants from Inserm, CNRS, Laboratoire d'Excellence consortium Inflamex (grant no. ANR-11-
IDEX-0005-02), ANR ANR-17-CE14-0002-01 (Diab1MAIT), the Fondation pour la Recherche Medicale (FRM grant nos DEQ20140329520, EQU201903007779), la Fondation Francophone pour la Recherche sur le Diabète to AL and Aide aux Jeunes Diabétiques fellowship to IN. MR and L. Bertrand were supported by the French Ministry of Research.

Authors' relationships and activities The authors declare that there are no relationships or activities that might bias, or be perceived to bias, their work.

Contribution statement IN and L. Beaudoin contributed to study conception and acquisition, analysis and interpretation of data. ZG, CR and PS acquired and analysed the data. MR performed most of the bioinformatic analyses. L. Bertrand contributed to interpretation of data. CB and EL recruited patients and provided intellectual input for conception and interpretation of data. IN, L. Beaudoin, MR, L. Bertrand and AL wrote the manuscript. AL supervised the work and brought substantial contributions to conception, analysis and interpretation of the data. All the authors revised the article critically for important intellectual content and finally approved the version to be published. AL is the guarantor of this work and, as such, had full access to all the data in the study and takes responsibility for the integrity of the data and the accuracy of the data analysis.

\section{References}

1. Atkinson MA, Eisenbarth GS, Michels AW (2014) Type 1 diabetes. Lancet 383(9911):69-82. https://doi.org/10.1016/S01406736(13)60591-7

2. DiMeglio LA, Evans-Molina C, Oram RA (2018) Type 1 diabetes. Lancet 391(10138):2449-2462. https://doi.org/10.1016/S01406736(18)31320-5

3. Ilonen J, Lempainen J, Veijola R (2019) The heterogeneous pathogenesis of type 1 diabetes mellitus. Nat Rev Endocrinol 15(11): 635-650. https://doi.org/10.1038/s41574-019-0254-y

4. Lehuen A, Diana J, Zaccone P, Cooke A (2010) Immune cell crosstalk in type 1 diabetes. Nat Rev Immunol 10(7):501-513. https://doi.org/10.1038/nri2787

5. Hull CM, Peakman M, Tree TIM (2017) Regulatory T cell dysfunction in type 1 diabetes: what's broken and how can we fix it? Diabetologia 60(10):1839-1850. https://doi.org/10.1007/s00125017-4377-1

6. Bluestone JA, Herold K, Eisenbarth G (2010) Genetics, pathogenesis and clinical interventions in type 1 diabetes. Nature 464(7293): 1293-1300. https://doi.org/10.1038/nature08933

7. Hughes JW, Riddlesworth TD, DiMeglio LA et al (2016) Autoimmune diseases in children and adults with type 1 diabetes from the T1D Exchange Clinic Registry. J Clin Endocrinol Metab 101(12):4931-4937. https://doi.org/10.1210/jc.2016-2478

8. Kahaly GJ, Hansen MP (2016) Type 1 diabetes associated autoimmunity. Autoimmun Rev 15(7):644-648. https://doi.org/10.1016/j. autrev.2016.02.017

9. Treiner E, Duban L, Bahram S et al (2003) Selection of evolutionarily conserved mucosal-associated invariant $\mathrm{T}$ cells by MR1. Nature 422(6928):164-169. https://doi.org/10.1038/nature01433

10. Ussher JE, Klenerman P, Willberg CB (2014) Mucosal-associated invariant T-cells: new players in anti-bacterial immunity. Front Immunol 5:450. https://doi.org/10.3389/fimmu.2014.00450 
11. Lantz O, Legoux F (2018) MAIT cells: an historical and evolutionary perspective. Immunol Cell Biol 96(6):564-572. https://doi.org/ 10.1111/imcb. 1034

12. Dusseaux M, Martin E, Serriari N et al (2011) Human MAIT cells are xenobiotic-resistant, tissue-targeted, CD161hi IL-17-secreting T cells. Blood 117(4):1250-1259. https://doi.org/10.1182/blood2010-08-303339

13. Toubal A, Nel I, Lotersztajn S, Lehuen A (2019) Mucosalassociated invariant $\mathrm{T}$ cells and disease. Nat Rev Immunol. https://doi.org/10.1038/s41577-019-0191-y

14. Nel I, Bertrand L, Toubal A, Lehuen A (2021) MAIT cells, guardians of skin and mucosa? Mucosal Immunol 1-12. https://doi.org/ 10.1038/s41385-021-00391-w

15. Corbett AJ, Eckle SBG, Birkinshaw RW et al (2014) T-cell activation by transitory neo-antigens derived from distinct microbial pathways. Nature 509(7500):361-365. https://doi.org/10.1038/ nature 13160

16. Ussher JE, Bilton M, Attwod E et al (2014) CD161++ CD8+ T cells, including the MAIT cell subset, are specifically activated by IL-12+IL-18 in a TCR-independent manner. Eur J Immunol 44(1): 195-203. https://doi.org/10.1002/eji.201343509

17. Leeansyah E, Svärd J, Dias J et al (2015) Arming of MAIT cell cytolytic antimicrobial activity is induced by IL-7 and defective in HIV-1 infection. PLoS Pathog 11(8):e1005072. https://doi.org/10. 1371/journal.ppat.1005072

18. Loh L, Wang Z, Sant S et al (2016) Human mucosal-associated invariant $\mathrm{T}$ cells contribute to antiviral influenza immunity via IL18-dependent activation. Proc Natl Acad Sci U S A 113(36): 10133-10138. https://doi.org/10.1073/pnas.1610750113

19. Pavlovic M, Gross C, Chili C, Secher T, Treiner E (2020) MAIT cells display a specific response to type 1 IFN underlying the adjuvant effect of TLR7/8 ligands. Front Immunol 11:2097. https://doi. org/10.3389/fimmu.2020.02097

20. Rouxel O, Da Silva J, Beaudoin L et al (2017) Cytotoxic and regulatory roles of mucosal-associated invariant $\mathrm{T}$ cells in type 1 diabetes. Nat Immunol 18(12):1321-1331. https://doi.org/10.1038/ni.3854

21. Le Bourhis L, Martin E, Péguillet I et al (2010) Antimicrobial activity of mucosal-associated invariant $\mathrm{T}$ cells. Nat Immunol 11(8):701-708. https://doi.org/10.1038/ni.1890

22. Rouxel O, Lehuen A (2018) Mucosal-associated invariant T cells in autoimmune and immune-mediated diseases. Immunol Cell Biol 96(6):618-629. https://doi.org/10.1111/imcb.12011

23. Gazali AM, Schroderus A-M, Näntö-Salonen K et al (2020) Mucosal-associated invariant T cell alterations during the development of human type 1 diabetes. Diabetologia 63(11):2396-2409. https://doi.org/10.1007/s00125-020-05257-7

24. Rouland M, Beaudoin L, Rouxel O et al (2021) Gut mucosa alterations and loss of segmented filamentous bacteria in type 1 diabetes are associated with inflammation rather than hyperglycaemia. Gut gutjnl-2020-323664. https://doi.org/10.1136/gutjnl-2020-323664

25. Battaglia M, Atkinson MA (2015) The streetlight effect in type 1 diabetes. Diabetes 64(4):1081-1090. https://doi.org/10.2337/db14-1208

26. Arif S, Leete P, Nguyen V et al (2014) Blood and islet phenotypes indicate immunological heterogeneity in type 1 diabetes. Diabetes 63(11):3835-3845. https://doi.org/10.2337/db14-0365

27. Leete P, Willcox A, Krogvold L et al (2016) Differential insulitic profiles determine the extent of $\beta$-cell destruction and the age at onset of type 1 diabetes. Diabetes 65(5):1362-1369. https://doi.org/ $10.2337 / \mathrm{db} 15-1615$

28. In't Veld P (2011) Insulitis in human type 1 diabetes: the quest for an elusive lesion. Islets 3(4):131-138. https://doi.org/10.4161/isl.3. 4.15728
29. Komulainen J, Kulmala P, Savola K et al (1999) Clinical, autoimmune, and genetic characteristics of very young children with type 1 diabetes. Diabetes Care 22(12):6

30. Karjalainen J, Salmela P, Ilonen J, Surcel HM, Knip M (1989) A comparison of childhood and adult type I diabetes mellitus. N Engl J Med 320(14):881-886. https://doi.org/10.1056/ NEJM198904063201401

31. Novak J, Dobrovolny J, Novakova L, Kozak T (2014) The decrease in number and change in phenotype of mucosal-associated invariant $\mathrm{T}$ cells in the elderly and differences in men and women of reproductive age. Scand J Immunol 80(4):271-275. https://doi.org/10. 1111/sji.12193

32. Chen P, Deng W, Li D et al (2019) Circulating mucosal-associated invariant $\mathrm{T}$ cells in a large cohort of healthy Chinese individuals from newborn to elderly. Front Immunol 10:260. https://doi.org/10. 3389/fimmu.2019.00260

33. Flament H, Rouland M, Beaudoin L et al (2021) Outcome of SARS-CoV-2 infection is linked to MAIT cell activation and cytotoxicity. Nat Immunol 22(3):322-335. https://doi.org/10.1038/ s41590-021-00870-Z

34. R Core Team (2021) R: a language and environment for statistical computing. R Foundation for Statistical Computing, Vienna, Austria

35. Reantragoon R, Corbett AJ, Sakala IG et al (2013) Antigen-loaded MR1 tetramers define $T$ cell receptor heterogeneity in mucosalassociated invariant T cells. J Exp Med 210(11):2305-2320. https://doi.org/10.1084/jem.20130958

36. Magalhaes I, Pingris K, Poitou C et al (2015) Mucosal-associated invariant $\mathrm{T}$ cell alterations in obese and type 2 diabetic patients. J Clin Invest 125(4):1752-1762. https://doi.org/10.1172/JCI78941

37. Carolan E, Tobin LM, Mangan BA et al (2015) Altered distribution and increased IL-17 production by mucosal-associated invariant T cells in adult and childhood obesity. J Immunol 194(12):57755780. https://doi.org/10.4049/jimmunol.1402945

38. Touch S, Assmann KE, Aron-Wisnewsky J et al (2018) Mucosalassociated invariant $\mathrm{T}$ (MAIT) cells are depleted and prone to apoptosis in cardiometabolic disorders. FASEB J fj201800052RR. https://doi.org/10.1096/fj.201800052RR

39. Reinert-Hartwall L, Honkanen J, Salo HM et al (2015) Th1/Th17 plasticity is a marker of advanced $\beta$ cell autoimmunity and impaired glucose tolerance in humans. J Immunol 194(1):68-75. https://doi.org/10.4049/jimmunol.1401653

40. Lee Y, Awasthi A, Yosef N et al (2012) Induction and molecular signature of pathogenic TH17 cells. Nat Immunol 13(10):991-999. https://doi.org/10.1038/ni.2416

41. $\mathrm{Hu} \mathrm{D}$, Notarbartolo S, Croonenborghs $\mathrm{T}$ et al (2017) Transcriptional signature of human pro-inflammatory T H 17 cells identifies reduced IL10 gene expression in multiple sclerosis. Nat Commun 8(1):1600. https://doi.org/10.1038/s41467-017-01571-8

42. Provine NM, Amini A, Garner LC et al (2021) MAIT cell activation augments adenovirus vector vaccine immunogenicity. Science 371(6528):521-526. https://doi.org/10.1126/science.aax8819

43. Diana J, Simoni Y, Furio L et al (2013) Crosstalk between neutrophils, B-1a cells and plasmacytoid dendritic cells initiates autoimmune diabetes. Nat Med 19(1):65-73. https://doi.org/10.1038/nm.3042

44. Whitacre CC (2001) Sex differences in autoimmune disease. Nat Immunol 2(9):777-780. https://doi.org/10.1038/ni0901-777

45. Beaudoin L, Laloux V, Novak J, Lucas B, Lehuen A (2002) NKT cells inhibit the onset of diabetes by impairing the development of pathogenic $\mathrm{T}$ cells specific for pancreatic beta cells. Immunity 17(6):725-736. https://doi.org/10.1016/s1074-7613(02)00473-9

Publisher's note Springer Nature remains neutral with regard to jurisdictional claims in published maps and institutional affiliations. 